\title{
H-AREA ACID/CAUSTIC BASIN GROUNDWATER MONITORING REPORT (U)
}

\section{THIRD QUARTER 1994}

\author{
Publication Date: December 1994
}

Authorized Derivative Classifier and Reviewing Official:

UNCLASSIFIED

Does Not Contain Unclassified

Controlled Nuclear Information

Westinghouse Savannah River Company

Savannah River Site

Aiken, SC 29808 

WSRC-TR-94-0491

Unclassified

\section{H-AREA ACID/CAUSTIC BASIN GROUNDWATER MONITORING REPORT (U)}

\section{THIRD QUARTER 1994}

Publication Date: December 1994

Authorized Derivative Classifier and Reviewing Official:

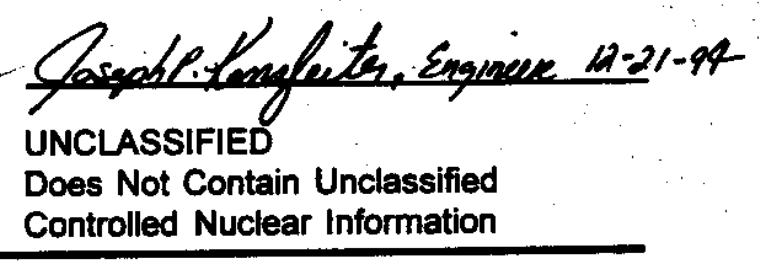

Westinghouse Savannah River Company Savannah River Site

Aiken, SC 29808

Prepared for the U.S. Department of Energy under Control Contract No. DE-AC09-89SR18035 


\section{Abstract}

During third quarter 1994, samples collected from the four. HAC monitoring wells at the H-Area Acid/Caustic Basin were analyzed for selected heavy metals, herbicides/pesticides, indicator parameters, major ions, radionuclide indicators, and other constituents. Monitoring results that exceeded the final Primary Drinking Water Standards (PDWS) or the Savannah River Site (SRS) flagging criteria or turbidity standard during third quarter are the focus of this report.

Tritium exceeded the final PDWS in all four HAC wells during third quarter 1994. Carbon tetrachloride exceeded the final PDWS in well HAC 4. Aluminum exceeded its Flag 2 criterion in all four HAC wells. Iron was elevated in wells HAC 1, 2, and 3. Manganese exceeded its Flag 2 criterion in well HAC 3, and total organic halogens was elevated in well HAC 2. No well samples exceeded the SRS turbidity standard.

Groundwater flow direction in the water table beneath the H-Area Acid/Caustic Basin was to the northwest during third quarter 1994. This data is consistent with previous quarters, when the flow direction has been to the northwest or the north-northwest. 
THIS PAGE LEFT BLANK INTENTIONALLY. 


\section{Contents}

Page

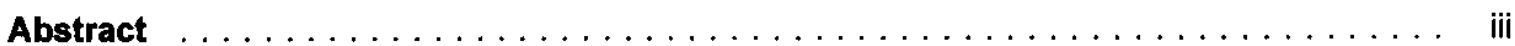

List of Figures $\ldots \ldots \ldots \ldots \ldots \ldots \ldots \ldots \ldots \ldots \ldots \ldots \ldots \ldots \ldots \ldots \ldots$

List of Tables $\ldots \ldots \ldots \ldots \ldots \ldots \ldots \ldots \ldots \ldots \ldots \ldots \ldots \ldots \ldots \ldots \ldots$

Executive Summary $\ldots \ldots \ldots \ldots \ldots \ldots \ldots \ldots \ldots \ldots \ldots \ldots \ldots \ldots$

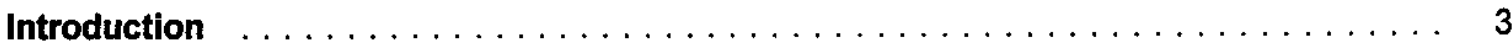

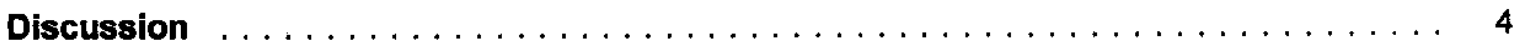

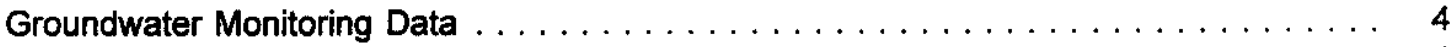

Analytical Results Exceeding Standards $\ldots \ldots \ldots \ldots \ldots \ldots \ldots \ldots \ldots \ldots \ldots \ldots$

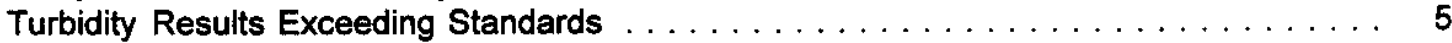

Water Elevations, Flow Directions, and Flow Rates $\ldots \ldots \ldots \ldots \ldots \ldots \ldots \ldots$

Results for Upgradient vs. Downgradient Wells $\ldots \ldots \ldots \ldots \ldots \ldots \ldots \ldots$

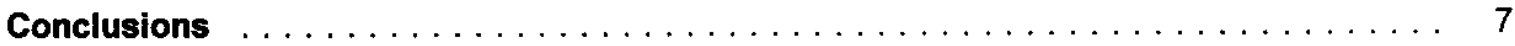

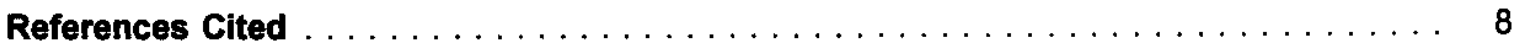

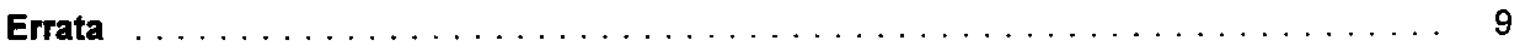

Appendix A-Final Primary Drinking Water Standards $\ldots \ldots \ldots \ldots \ldots \ldots \ldots$

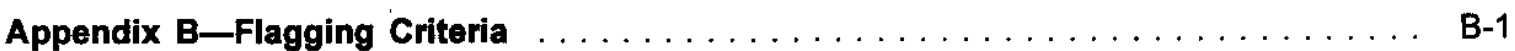

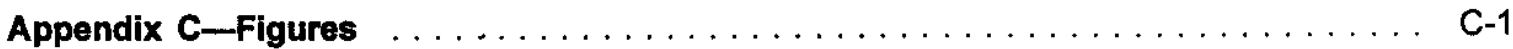

Appendix D-Groundwater Monitoring Results Tables $\ldots \ldots \ldots \ldots \ldots \ldots \ldots$

Appendix E-Data Quality/Usability Assessment $\ldots \ldots \ldots \ldots \ldots \ldots \ldots \ldots$ 


\section{List of Figures}

Page

1. Location of the H-Area Acid/Caustic Basin at the Savannah River Site $\ldots \ldots \ldots$ C-3

2. Location of Groundwater Monitoring Wells at the H-Area Acid/Caustic Basin . . . . . C-4

3. Piezometric Surface Map of the Water Table at the H-Area Acid/Caustic Basin . . . C-5

\section{List of Tables}

Page

1. Maximum Results for Constituents Exceeding Final Primary Drinking Water

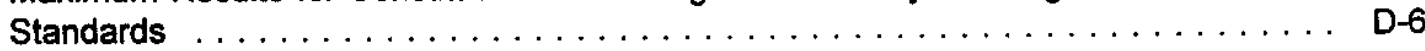

2. Maximum Results for Constituents Exceeding Other Flag 2 Criteria or the SRS

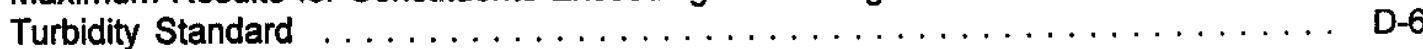

3. Groundwater Monitoring Results for Individual Wells $\ldots \ldots \ldots \ldots \ldots \ldots \ldots$ D-7 


\section{Executive Summary}

The four monitoring wells at the H-Area Acid/Caustic Basin are sampled quarterly as part of the Savannah River Site (SRS) Groundwater Monitoring Program and to comply with a consent decree signed May 26, 1988, by the U.S. District Court (District of South Carolina, Aiken Division).

During third quarter 1994, groundwater from the HAC wells was analyzed for selected heavy metals, herbicides/pesticides, indicator parameters, major ions, radionuclide indicators, and other constituents. Monitoring results that exceeded the final Primary Drinking Water Standards (PDWS), the SRS flagging criteria, or the SRS turbidity standard are the focus of this report.

During third quarter 1994, tritium exceeded the final PDWS in all four HAC wells, with activities from 3.3E+01 to $6.5 E+01 \mathrm{pCi} / \mathrm{mL}$. Carbon tetrachloride exceeded the final PDWS in well HAC 4, with a concentration of $8.5 \mu \mathrm{g} / \mathrm{L}$. Aluminum exceeded its Flag 2 criterion in all four HAC wells, ranging from 64 to $303 \mu \mathrm{g} / \mathrm{L}$. Iron exceeded its Flag 2 criterion in wells HAC 1, 2, and 3. Manganese was elevated in well HAC 3 . Total organic halogens exceeded its Flag 2 criterion in well HAC 2. No well samples exceeded the SRS turbidity standard.

Groundwater flow direction in the water table beneath the H-Area Acid/Caustic Basin was to the northwest during third quarter 1994. During the last two years, the groundwater flow direction has been consistently to the northwest or the north-northwest. An apparent change in flow direction during second quarter 1994 was attributed to the lack of water elevations for wells HTF 16 and 17 and the anomalous water elevation reported for well HAC 2 during second quarter. During third quarter, flow direction was again consistent with historical trends. 
THIS PAGE LEFT BLANK INTENTIONALLY. 


\section{Introduction}

The H-Area Acid/Caustic Basin is southwest of the H-Area Canyon Building and north of the H-Area Tank Farm at the Savannah River Site (SRS) (Figure 1, Appendix C). The following description outlines important events at the H-Area Acid/Caustic Basin.

- The basin, constructed in the early 1950 s, is an unlined earthen pit that received dilute sulfuric acid and sodium hydroxide solutions and other wastes from several areas within SRS. The basin provided an area for the mixing and neutralization of the dilute solutions before their discharge into nearby streams (Heffner and Exploration Resources, 1991).

- Disposal of acid/caustic solutions to the H-Area Acid/Caustic Basin was discontinued in 1982; however, the basin received steam condensate from a hose box and drainage from a chemical pad until 1985 (Heffner and Exploration Resources, 1991).

- Under the terms of a consent decree signed May 26, 1988, by the U.S. District Court (Civil Action 1:85-2583-6, District of South Carolina, Aiken Division), the basin became subject to requirements of Subtitle $C$ of the Resource Conservation and Recovery Act (RCRA), the South Carolina Hazardous Waste Management Regulations (SCHWMR), and associated regulations on June 1, 1988.

- In the summer of 1988, a network of monitoring wells was proposed for the basin to ensure compliance with SCHWMR; in August 1988, four monitoring wells, HAC 1, 2, 3, and 4, were installed at the H-Area Acid/Caustic Basin (EPD/EMS, 1994) (Figure 2, Appendix C).

- The revised Groundwater Quality Assessment Plan (WSRC, 1991), submitted to the South Carolina Department of Health and Environmental Control (SCDHEC) on April 30, 1991, indicated that the monitoring well network at the H-Area Acid/Caustic Basin is sufficient to detect any degradation of the groundwater due to past operations at the basin.

- During July through September 1993, with SCDHEC's permission to proceed at risk, SRS stabilized the $\mathrm{H}$ - and P-Area Acid/Caustic Basins as proposed in the Interim Status Closure Plan for the F-, H-, K-, and P-Area Acid/Caustic Basins (Revision 3, February 5, 1992). The basins were dewatered and filled with compacted clay-rich soil, and a vegetative cover of winter- and drought-hardy grass was established.

Each quarter, the Environmental Protection Department/Environmental Monitoring Section (EPD/ EMS) samples the monitoring wells at the H-Area Acid/Caustic Basin as part of the SRS Groundwater Monitoring Program. The Environmental Restoration Department provides a quarterly report describing the monitoring results to SCDHEC in compliance with SCHWMR. 


\section{Discussion}

\section{Groundwater Monitoring Data}

The groundwater sampling procedure (EPD/EMS, 1992) requires evacuation of a minimum of two well volumes and stabilization of $\mathrm{pH}$, specific conductance, and turbidity prior to sample collection. Stability is established when a minimum of three successive measurements, taken within a given time period, are within a specified tolerance range. If a well pumps dry before two well volumes are purged or before stabilization is achieved, it must be revisited within 24 hours for the data to be considered from a single sampling event. On the second visit within 24 hours, samples are taken without purging or stability measurements; thus, these samples may not be representative of the groundwater quality.

During third quarter 1994, samples from the four monitoring wells at the H-Area Acid/Caustic Basin were analyzed for selected heavy metals, herbicides/pesticides, indicator parameters, major ions, radionuclide indicators, and other constituents. This report describes monitoring results that exceeded the Safe Drinking Water Act final Primary Drinking Water Standards (PDWS) or screening levels set by the U.S. Environmental Protection Agency (EPA) (Appendix A); the South Carolina final PDWS for lead (Appendix A); other SRS Flag 2 criteria based on final and proposed PDWS, Secondary Drinking Water Standards, and method detection limits (Appendix B); or the SRS turbidity standard. Constituent levels that equal or exceed the final PDWS, screening levels, or Flag 2 criteria are described as exceeding standards, above standards, or as elevated.

The final PDWS for individual analytes provided in Appendix A may not always match the SRS flagging criteria provided in Appendix B. The final PDWS are used as guidelines in this compliance report to meet regulatory requirements; the flagging criteria are used by the Environmental Protection Department/Environmental Monitoring Section to identify relative levels of constituents in the groundwater and as guides for scheduling groundwater sampling.

\section{Analytical Results Exceeding Standards}

Results for analytes that exceeded the final PDWS (see Appendix A) during third quarter 1994 are summarized in Table 1 (Appendix D). All four HAC wells contained tritium activities that exceeded the final PDWS, with activities ranging from $3.3 \mathrm{E}+01$ to $6.5 \mathrm{E}+01 \mathrm{pCi} / \mathrm{mL}$. Carbon tetrachloride exceeded the final PDWS in well HAC 4, with a concentration of $8.5 \mu \mathrm{g} / \mathrm{L}$.

Constituents that exceeded other Flag 2 criteria (see Appendix B) during third quarter 1994 are summarized in Table 2 (Appendix D). Aluminum, which was added to the analytes included in comprehensive analyses beginning first quarter 1993, exceeded its Flag 2 criterion in all four HAC wells, with a maximum concentration of $303 \mu \mathrm{g} / \mathrm{L}$ in well HAC 3. 'Iron exceeded its Flag 2 criterion in wells HAC 1,2 , and 3 , with a maximum concentration of $1,280 \mu \mathrm{g} / \mathrm{L}$ in HAC 2. Manganese exceeded its Flag 2 criterion in well $\mathrm{HAC} \mathrm{3}$, with a concentration of $61 \mu \mathrm{g} / \mathrm{L}$. Total organic halogens also exceeded its Flag 2 criterion in well HAC 2, with a concentration of $110 \mu \mathrm{g} / \mathrm{L}$.

Table 3 (Appendix D) presents all of the results for individual wells and indicates the analytical laboratories that conducted the analyses, the dilution factors used in the analyses, and the analyses that received modifiers (which help identify laboratory accuracy and precision) or that ex- 
ceeded the EPA-approved holding times during third quarter 1994. Constituent results in Table 3 that appear to equal the final PDWS but are not marked in the ST column (exceeded final PDWS or screening level) are below the final PDWS in the database. Database results, the results that are compared to the final PDWS, are entered with more significant digits than the results given in this report. Apparent discrepancies are the result of the rounding of reported results.

Table 3 also lists the number of well volumes purged from each well during third quarter 1994 at the H-Area Acid/Caustic Basin. Wells HAC 2 and 3 went dry during purging; thus, they may not have produced representative groundwater samples.

Appendix $D$ provides definitions of the abbreviations and the modifiers used in the results tables as well as descriptions of holding times, data rounding, and data qualification practices.

Appendix $E$ provides a general assessment of the quality and usability of the data provided by EPD/EMS.

\section{Turbidity Results Exceeding Standards}

The value of 5 nephelometric turbidity units (NTU), established by EPA (1986) as a general standard for acceptability of groundwater samples, is considered unrealistic for monitoring wells at SRS. Gass (1989) has documented turbidity measurements ranging up to 5,000 NTU from properly designed wells screened in poorly productive formations, such as those screened in the water table. During the 1989 RCRA Compliance Evaluation Inspection, officials from EPA Region IV indicated that the SRS turbidity standard of 50 NTU is conservative. These officials also agreed that water-table wells in this area often screen nonaquifer formations, rendering development of these wells more difficult due to the low yield and high proportion of mobile fines typical of these formations (Bergren and Bennett, 1989).

During third quarter 1994, none of the samples exceeded the SRS turbidity standard of 50 NTU (Table 3, Appendix D).

\section{Water Elevations, Flow Directions, and Flow Rates}

Water-table elevations and the groundwater flow direction beneath the H-Area Acid/Caustic Basin are shown in Figure 3 (Appendix C). The horizontal gradient at the H-Area Acid/Caustic Basin is very low. Water elevations from nine nearby wells of the HTF series were included in determining water-elevation contours to supply more complete information on groundwater movement beneath the H-Area Acid/Caustic Basin and facilitate the determination of local flow direction. The northwest groundwater flow direction (using universal transverse Mercator coordinates) was determined from this quarter's water-level elevations for wells $H A C$ 1, 2, 3, and 4 and adjacent wells HTF $13,14,15,16,17,18,19,20$, and 21 . The flow direction for the last two years has been consistently northwest or north-northwest. 
The groundwater flow rate in the water table (Aquifer Zone $\| \mathrm{B}_{2}$ ) beneath the $\mathrm{H}$-Area Acid/Caustic Basin is estimated using the following equation:

$$
\text { Flow }(\mathrm{ft} / \text { day })=\frac{\text { Hydraulic Conductivity }(\mathrm{ft} / \text { day) }}{\text { Porosity (unitless) }} \times \frac{d h(\mathrm{ft})}{d l(\mathrm{ft})}
$$

A hydraulic conductivity constant of $10 \mathrm{ft} /$ day (Geraghty \& Miller, 1990) is used as a conservative estimate (i.e., the actual hydraulic conductivity should be somewhat less than $10 \mathrm{ft} / \mathrm{day}$ ). The effective porosity value is estimated at 20 percent (Killian et al., 1987); $d h$ is the difference in head, and $d l$ is the length of the flow path to the nearest $10 \mathrm{ft}$. Flow rate estimates vary depending on the hydraulic gradient between wells, the size of the area under consideration, and the number of data points. For this reason, the estimation of flow rate should be considered accurate to an order of magnitude only.

Flow rate per day is calculated to two significant figures using the above equation. This value is then multiplied by 365 and rounded to two significant figures for the flow rate per year.

Using the above equation, with $d h=4 \mathrm{ft}$ and $d l=370 \mathrm{ft}$, the flow rate estimate for groundwater in the water table beneath the H-Area Acid/Caustic Basin (see Figure 3, Appendix $\mathrm{C}$ ) is as follows:

$$
\frac{10}{0.20} \times \frac{4}{370}=0.54 \mathrm{ft} / \mathrm{day}
$$

\section{$0.54 \mathrm{ft} /$ day $\times 365$ days $\approx 200 \mathrm{ft} /$ year}

\section{Results for Upgradient vs. Downgradient Wells}

Well HAC 4 is the upgradient well, and wells $\operatorname{HAC} 1,2$, and 3 are the downgradient wells at the H-Area Acid/Caustic Basin.

During third quarter 1994, aluminum, carbon tetrachloride, and tritium were elevated in the upgradient well.

Tritium also exceeded the final PDWS in all three downgradient wells, with activity in well HAC 1 approximately twice that detected in the other HAC wells. Aluminum exceeded its Flag 2 criterion in all three downgradient wells. Iron, not detected above standards in the upgradient well, exceeded the Flag 2 criterion in all downgradient wells. Manganese was elevated in downgradient well $\mathrm{HAC} 3$, and total organic halogens was elevated in downgradient well HAC 2. 


\section{Conclusions}

Tritium activities exceeded the final PDWS during third quarter 1994 in all four HAC wells, with activities from $3.3 \mathrm{E}+01$ to $6.5 \mathrm{E}+01 \mathrm{pCi} / \mathrm{mL}$. Because historical records indicate that no radionuclides were disposed of at this waste management unit (Heffner and Exploration Resources, 1991), elevated levels of tritium in the HAC wells are not considered a result of seepage from the acid/caustic basin. Other facilities within $\mathrm{H}$ Area, including the high-level-waste tank farm adjacent to the H-Area Acid/Caustic Basin, are possible sources of the tritium.

Carbon tetrachloride exceeded the final PDWS in well HAC 4, with a concentration of $8.5 \mu \mathrm{g} / \mathrm{L}$. Because well HAC 4 is upgradient of the H-Area Acid/Caustic Basin, the source of the carbon tetrachloride is not the basin. Heptachlor epoxide, which exceeded final PDWS in well HAC 4 during first quarter 1994, was not analyzed in the HAC wells during third quarter.

Aluminum exceeded the Flag 2 criterion in all four HAC wells. Iron exceeded the Flag 2 criterion in all downgradient wells, and manganese exceeded the Flag 2 criterion in downgradient well HAC 3. Total organic halogens exceeded the Flag 2 criterion in downgradient well HAC 2. Generally, elevated levels of constituents found in downgradient wells but not in upgradient wells at a waste management unit are considered products of the waste management unit.

No well samples exceeded the 50 NTU SRS turbidity standard.

Third quarter 1994 water-table elevations at the H-Area Acid/Caustic Basin indicate that groundwater flow was toward the northwest at a rate of approximately $200 \mathrm{ft} / \mathrm{year}$. The flow direction has been consistently northwest or north-northwest for the last two years. Lack of water elevations from wells HTF 16 and 17 and an anomalous water elevation from well HAC 2 contributed to an apparent change in flow direction to the west during second quarter 1994. Third quarter groundwater flow direction and rate were again consistent with historical trends.

The revised Groundwater Quality Assessment Plan (WSRC, 1991) for the unit provides evidence that wells $\mathrm{HAC} 1,2$, and 3 are consistently downgradient of well HAC 4 and that the monitoring well network is sufficient to detect degradation of the groundwater due to past operations at the basin. 
WSRC-TR-94-0491

Unclassiffed

\section{References Cited}

Bergren, C. L., and C. B. Bennett, 1989. Assessment of SRS Groundwater Monitoring Wells Impacted by Turbidity, WSRC-RP-89-891. Westinghouse Savannah River Company, Savannah River Site, Aiken, SC.

EPA (U.S. Environmental Protection Agency), 1986. RCRA Ground Water Monitoring Technical Enforcement Guidance Document, OSWER-9950.1. Washington, DC.

EPD/EMS (Environmental Protection Department/Environmental Monitoring Section), 1992. Hydrogeologic Data Collection Procedures and Specifications: Sampling Groundwater Monitoring Wells, Manual 3Q5, Chapter 14, Revision 0. Westinghouse Savannah River Company, Savannah River Site, Aiken, SC.

EPD/EMS (Environmental Protection Department/Environmental Monitoring Section), 1994. Environmental Protection Department's Well Inventory (through the second quarter of 1994), ESH-EMS-940518. Westinghouse Savannah River Company, Savannah River Site, Aiken, SC.

Gass, T. E., 1989. Monitoring Wells in Non-Aquifer Formations. Water Well Journal, 43(2):27-29.

Geraghty \& Miller, Inc., 1990. Evaluation of Integrated Waste Facility Closure Capping on Ground-Water Flow and Solute Transport in General Separations Area, Savannah River Site: Flow Model and Particle-Tracking Analysis, Final Report. Prepared by Geraghty \& Miller Modeling Group for Westinghouse Savannah River Company, Waste Management Technology, Savannah River Site, Aiken, SC.

Heffner, J. D., and Exploration Resources, Inc., 1991. Technical Summary of Groundwater Quality Protection Program at the Savannah River Site (1952-1986), Volume L-Site Geohydrology and Waste Sites, DPSP-88-1002. Westinghouse Savannah River Company, Savannah River Site, Aiken, SC.

Killian, T. H., N. L. Kolb, P. Corbo, and I. W. Marine, 1987. F-Area Seepage Basins, DPST-85-704. E. I. du Pont de Nemours \& Company, Savannah River Laboratory, Aiken, SC.

WSRC (Westinghouse Savannah River Company), 1991. F-, H-, K-, and P-Area Acid/Caustic Basins Groundwater Quality Assessment Plan, WSRC-TR-91-178, Revision 1.0. Westinghouse Savannah River Company, Savannah River Site, Aiken, SC.

WSRC, 1992. F-, H-, K-, and P-Area Acid/Caustic Basins Interim Status Closure Plan, Revision 3. Westinghouse Savannah River Company, Savannah River Site, Aiken, SC. 
WSRC-TR-94-0491

Unclassiffed

\section{Errata}

In tables with four quarters of data, some values for earlier quarters may differ from values for those same quarters presented in earlier reports because some reanalyses may have been performed by the laboratories after the reports were printed.

Third Quarter 1993 through Fourth Quarter 1993:

- No errata have been reported.

First Quarter 1994:

- Page 4, Groundwater Monitoring Data: The copper standard is the final PDWS established by EPA.

Second Quarter 1994:

- No errata have been reported. 
WSRC-TR-94-0491

Unclassified

THIS PAGE LEFT BLANK INTENTIONALLY. 


\section{Appendix A}

\section{Final Primary Drinking Water Standards}


WSRC-TR-94-0491

Unclassified

THIS PAGE LEFT BLANK INTENTIONALLY. 
Final Primary Drinking Water Standards

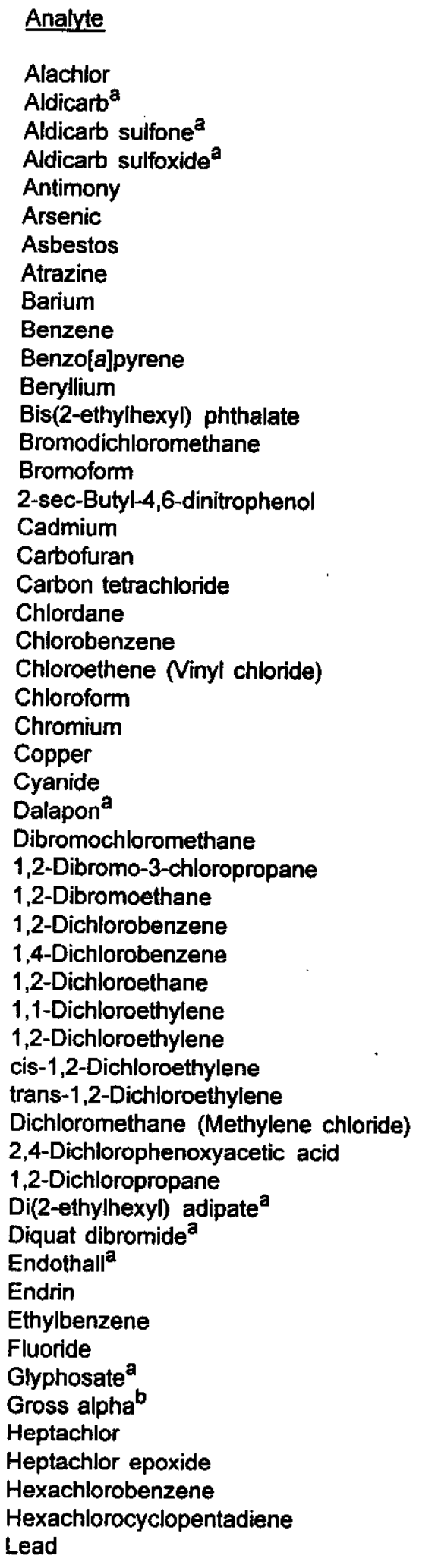

\begin{tabular}{|c|c|c|}
\hline Unit & Level & Status \\
\hline$\mu g / L$ & 2 & Final \\
\hline$\mu g / L$ & 3 & Final \\
\hline$\mu g / L$ & 2 & Final \\
\hline$\mu g / L$ & 4 & Final \\
\hline$\mu \mathrm{g} / \mathrm{L}$ & 6 & Final \\
\hline$\mu g / L$ & 50 & Final \\
\hline Fibers $/ L$ & $7,000,000$ & Final \\
\hline$\mu g / L$ & 3 & Final \\
\hline$\mu g / L$ & 2,000 & Final \\
\hline$\mu g / h$ & 5 & Final \\
\hline$\mu g / L$ & 0.2 & Final \\
\hline$\mu g / L$ & 4 & Final \\
\hline$\mu g / L$ & 6 & Final \\
\hline$\mu g / L$ & 100 & Final \\
\hline$\mu \mathrm{g} / \mathrm{L}$ & 100 & Final \\
\hline$\mu g / L$ & 7 & Final \\
\hline$\mu g / L$ & 5 & Final \\
\hline$\mu g / L$ & 40 & Final \\
\hline$\mu g / L$ & 5 & Final \\
\hline$\mu \mathrm{g} / \mathrm{L}$ & 2 & Final \\
\hline$\mu g / L$ & 100 & Final \\
\hline$\mu g / L$ & 2 & Final \\
\hline$\mu g / L$ & 100 & Final \\
\hline$\mu g / L$ & 100 & Final \\
\hline$\mu g /$ & 1,300 & Final \\
\hline$\mu g / L$ & 200 & Final \\
\hline$\mu g / L$ & 200 & Final \\
\hline$\mu g / L$ & 100 & Final \\
\hline$\mu g / L$ & 0.2 & Final \\
\hline$\mu \mathrm{g} / \mathrm{L}$ & 0.05 & Final \\
\hline$\mu g / L$ & 600 & Final \\
\hline$\mu g / L$ & 75 & Final \\
\hline$\mu g / L$ & 5 & Final \\
\hline$\mu g / h$ & 7 & Final \\
\hline$\mu g / \mathrm{L}$ & 50 & Final \\
\hline$\mu g / h$ & 70 & Final \\
\hline$\mu g / L$ & 100 & Final \\
\hline$\mu g / L$ & 5 & Final \\
\hline$\mu g / L$ & 70 & Final \\
\hline$\mu g / L$ & 5 & Final \\
\hline$\mu g / L$ & 400 & Final \\
\hline$\mu g / L$ & 20 & Final \\
\hline$\mu g / L$ & 100 & Final \\
\hline$\mu g / L$ & 2 & Final \\
\hline$\mu g / L$ & 700 & Final \\
\hline$\mu g / L$ & 4,000 & Final \\
\hline$\mu g / L$ & 700 & Final \\
\hline $\mathrm{pCi} / \mathrm{L}$ & $1.5 \mathrm{E}+01$ & Final \\
\hline$\mu \mathrm{g} / \mathrm{L}$ & 0.4 & Final \\
\hline$\mu g / L$ & 0.2 & Final \\
\hline$\mu g / L$ & 1 & Final \\
\hline$\mu g / L$ & 50 & Final \\
\hline$\mu g / L$ & 50 & Final \\
\hline
\end{tabular}

Source

EPA, 1993

EPA, 1993

EPA, 1993

EPA, 1993

EPA, 1993

EPA, 1993

EPA, 1993

EPA, 1993

EPA, 1993

EPA, 1993

EPA, 1993

EPA, 1993

EPA, 1993

EPA, 1993

EPA, 1993

EPA, 1993

EPA, 1993

EPA, 1993

EPA, 1993

EPA, 1993

EPA, 1993

EPA, 1993

EPA, 1993

EPA, 1993

EPA, 1993

EPA, 1993

EPA, 1993

EPA, 1993

EPA, 1993

EPA, 1993

EPA, 1993

EPA, 1993

EPA, 1993

EPA, 1993

EPA, 1993

EPA, 1993

EPA, 1993

EPA, 1993

EPA, 1993

EPA, 1993

EPA, 1993

EPA, 1993

EPA, 1993

EPA, 1993

EPA, 1993

EPA, 1993

EPA, 1993

EPA, 1993

EPA, 1993

EPA, 1993

EPA, 1993

EPA, 1993

SCDHEC, 1981 


\begin{tabular}{|c|c|c|c|c|}
\hline Analyte & Unit & Level & Status & Source \\
\hline Lindane & $\mu g / h$ & 0.2 & Final & EPA, 1993 \\
\hline Mercury & $\mu g / L$ & 2 & Final & EPA, 1993 \\
\hline Methoxychlor & $\mu g / L$ & 40 & Final & EPA, 1993 \\
\hline Nickel & $\mu g / L$ & 100 & Final & EPA, 1993 \\
\hline Nitrate as nitrogen & $\mu g / L$ & 10,000 & Final & EPA, 1993 \\
\hline Nitrate-nitrite as nitrogen & $\mu g / L$ & 10,000 & Final & EPA, 1993 \\
\hline Nitrite as nitrogen & $\mu g / L$ & 1,000 & Final & EPA, 1993 \\
\hline Nonvolatile beta & pCin & $5 E+01$ & Interim Final & EPA, 1977 \\
\hline Oxamyl ${ }^{\mathrm{a}}$ & $\mu \mathrm{g} / \mathrm{L}$ & 200 & Final & EPA, 1993 \\
\hline PCB 1016 & $\mu \mathrm{g} / \mathrm{L}$ & 0.5 & Final & EPA, 1993 \\
\hline РCB 1221 & $\mu \mathrm{g} / \mathrm{L}$ & 0.5 & Final & EPA, 1993 \\
\hline РCB 1232 & $\mu g / L$ & 0.5 & Final & EPA, 1993 \\
\hline PCB 1242 & $\mu g / L$ & 0.5 & Final & EPA, 1993 \\
\hline РСВ 1248 & $\mu g / L$ & 0.5 & Final & EPA, 1993 \\
\hline РСВ 1254 & $\mu \mathrm{g} / \mathrm{L}$ & 0.5 & Final & EPA, 1993 \\
\hline PCB 1260 & $\mu g / L$ & 0.5 & Final & EPA, 1993 \\
\hline РСВ 1262 & $\mu g / L$ & 0.5 & Final & EPA, 1993 \\
\hline Pentachlorophenol & $\mu g / L$ & 1 & Final & EPA, 1993 \\
\hline Picloram ${ }^{a}$ & $\mu g /$ & 500 & Final & EPA, 1993 \\
\hline Selenium & $\mu g / L$ & 50 & Final & EPA, 1993 \\
\hline Simazine $^{a}$ & $\mu g / L$ & 4 & Final & EPA, 1993 \\
\hline Strontium-89/90 & $\mathrm{pCi} / \mathrm{L}$ & $8 \mathrm{E}+00$ & Final & EPA, 1993 \\
\hline Strontium-90 & $\mathrm{pCin}$ & $8 \mathrm{E}+00$ & Final & EPA, 1993 \\
\hline Styrene & $\mu g / h$ & 100 & Final & EPA, 1993 \\
\hline 2,3,7,8-TCDD & $\mu g / L$ & 0.00003 & Final & EPA, 1993 \\
\hline Tetrachloroethylene & $\mu g / L$ & 5 & Final & EPA, 1993 \\
\hline Thallium & $\mu g / L$ & 2 & Final & EPA, 1993 \\
\hline Toluene & $\mu g / L$ & 1,000 & Final & EPA, 1993 \\
\hline Toxaphene & $\mu g / L$ & 3 & Final & EPA, 1993 \\
\hline 2,4,5-TP (Silvex) & $\mu g / L$ & 50 & Final & EPA, 1993 \\
\hline 1,2,4-Trichlorobenzene & $\mu g /$ & 70 & Final & EPA, 1993 \\
\hline 1,1,1-Trichloroethane & $\mu g / L$ & 200 & Final & EPA, 1993 \\
\hline 1,1,2-Trichloroethane & $\mu g / \mathrm{L}$ & 5 & Final & EPA, 1993 \\
\hline Trichloroethylene & $\mu g / L$ & 5 & Final & EPA, 1993 \\
\hline Tritium & $\mathrm{pCi} / \mathrm{mL}$ & $2 E+01$ & Final & EPA, 1993 \\
\hline Xylenes & $\mu g / L$ & 10,000 & Final & EPA, 1993 \\
\hline
\end{tabular}

Note: Final PDWS were assigned to alachlor, aldicarb, aldicarb sulfone, aldicarb sulfoxide, atrazine, carbofuran, dalapon, di(2-ethylhexyl) adipate, diquat dibromide, endothall, glyphosate, oxamyl, picloram, and simazine in the SRS Groundwater Monitoring Program for the first time beginning first quarter 1994.

a At present, EMS does not perform this analysis because the constituent is not in the current contract.

b The standard given is for gross alpha including radium-226 but excluding radon and uranium.

c For double radionuclide analyses where each separate radionuclide has its own standard, the more stringent standard is used. 


\section{References}

EPA (U.S. Environmental Protection Agency), 1977. National Interim Primary Drinking Water Regulations, EPA-570/9-76-003. Washington, DC.

EPA (U.S. Environmental Protection Agency), 1993. National Primary Drinking Water Regulations, Code of Federal Regulations, Title 40, Part 141, pp. 592-732. Washington, DC.

SCDHEC (South Carolina Department of Health and Environmental Control), 1981. State Primary Drinking Water Regulations, R.61-58.5. Columbia, SC. 
THIS PAGE LEFT BLANK INTENTIONALLY. 


\section{Appendix B}

\section{Flagging Criteria}


THIS PAGE LEFT BLANK INTENTIONALLY. 


\section{Flagging Criteria}

The Savannah River Site Environmental Protection Department/Environmental Monitoring Section (EPD/EMS) flagging criteria are as follows:

- Flag 2. criteria for constituents equal the Safe Drinking Water Act (SDWA) final Primary Drinking Water Standards (PDWS), the SDWA proposed PDWS, or the SDWA Secondary Drinking Water Standards (SDWS). If a constituent does not have a drinking water standard, the Flag 2 criterion equals 10 times the method detection limit (MDL) calculated as the 90th percentile detection limit obtained recently by one of the primary analytical laboratories.

- Flag 1 criteria for constituents equal one-half of the final PDWS, one-half the proposed PDWS, or one-half the SDWS. If a constituent does not have a drinking water standard, the Flag 1 criterion equals 5 times the MDL calculated as the 90th percentile detection limit obtained recently by one of the primary analytical laboratories.

- Flag 0 criteria are assigned to constituent levels below Flag 1 criteria, constituent levels below the sample detection limits, or constituents having no flagging criteria.

The following parameters are exceptions to the flagging rules:

- EPD/EMS sets flagging criteria for $\mathrm{pH}$ and specific conductance. No flags are set for alkalinity, calcium, carbonate, magnesium, potassium, silica, sodium, total dissolved solids, total phosphates (as P), and total phosphorus. Analyses for these parameters are conducted as part of the biennial comprehensive analyses or by special request.

- Aesthetic parameters such as color, corrosivity, Eh, odor, surfactants, and turbidity are not assigned flagging criteria but are analyzed by special request.

- Common laboratory contaminants and cleaners such as dichloromethane (methylene chloride), ketones, phthalates, and toluene are not assigned flagging criteria unless they have primary drinking water standards. These constituents are analyzed by special request.

\begin{tabular}{|c|c|c|c|c|}
\hline Analyte & Unit & Flag 1 & Flag 2 & Source $^{a}$ \\
\hline Acenaphthene & $\mu g / L$ & 50 & 100 & EPA Method 8270 \\
\hline Acenaphthylene & $\mu g / L$ & 50 & 100 & EPA Method 8270 \\
\hline Acetone & $\mu g / L$ & 500 & 1,000 & EPA Method 8240 \\
\hline Acetonitrile (Methyl cyanide) & $\mu g / L$ & 500 & 1,000 & EPA Method 8240 \\
\hline Acetophenone & $\mu g / L$ & 50 & 100 & EPA Method 8270 \\
\hline 2-Acetylaminofluorene & $\mu g / L$ & 50 & 100 & EPA Method 8270 \\
\hline Acrolein & $\mu g / L$ & 100 & 200 & EPA Method 8240 \\
\hline Acrylonitrile & $\mu g / L$ & 100 & 200 & EPA Method 8240 \\
\hline Actinium-228 & $\mathrm{pCi} / \mathrm{L}$ & $1.64 \mathrm{E}+03$ & $3.27 E+03$ & Proposed PDWS (EPA, 1991) \\
\hline Alachlor & $\mu \mathrm{g} / \mathrm{h}$ & 1 & 2 & Final PDWS (EPA, 1993a) \\
\hline Aldicarb ${ }^{b}$ & $\mu g / h$ & 1.5 & 3 & Final PDWS (EPA, 1993a) \\
\hline Aldicarb sulfone $e^{b}$ & $\mu g / L$ & 1 & 2 & Final PDWS (EPA, 1993a) \\
\hline Aldicarb sulfoxide ${ }^{b}$ & $\mu g / L$ & 2 & 4 & Final PDWS (EPA, 1993a) \\
\hline Aldrin & $\mu g / L$ & 0.25 & 0.5 & EPA Method 8080 \\
\hline Alkalinity (as $\mathrm{CaCO}_{3}$ ) & & No flag & No flag & Set by EPD/EMS \\
\hline Allyl chloride & $\mu g /$ & 250 & 500 & EPA Method 8240 \\
\hline Aluminum & $\mu g / L$ & 25 & 50 & SDWS (EPA, 1993b) \\
\hline Aluminum, dissolved & $\mu g / L$ & 25 & 50 & SDWS (EPA, 1993b) \\
\hline Aluminum, total recoverable & $\mu g / L$ & 25 & 50 & SDWS (EPA, 1993b) \\
\hline
\end{tabular}


Analyte

Americium-241

Americium-243

4-Aminobiphenyl

Ammonia

Ammonia nitrogen

Aniline

Anthracene

Antimony

Antimony, dissolved

Antimony, total recoverable

Antimony-125

Aramite

Arsenic

Arsenic, dissolved

Arsenic, total recoverable

Asbestos

Atrazine

Azobenzene

Barium

Barium, dissolved

Barium, total recoverable

Barium-140 ${ }^{\mathrm{C}}$

Benzene

alpha-Benzene hexachloride

beta-Benzene hexachloride

delta-Benzene hexachloride

Benzidine

Benzo[a]anthracene

Benzo[b]fluoranthene

Benzo[k]fluoranthene

Benzoic acid

Benzo[g,h,iperylene

Benzo[a]pyrene

1,4-Benzoquinone

Benzyl alcohol

Beryllium

Beryllium, dissolved

Beryllium, total recoverable

Beryllium-7

Bis(2-chloroethoxy) methane

Bis(2-chloroethyl) ether

Bis(2-chloroisopropyl) ether

Bis(chloromethyl) ether

Bis(2-ethylhexyl) phthalate

Bismuth-214

Boron

Boron, dissolved

Boron, total recoverable

Bromide

Bromodichloromethane

Bromoform

Bromomethane (Methyl bromide)

4-Bromophenyl phenyl ether

Butylbenzyl phthalate

2-sec-Butyl-4,6-dinitrophenol

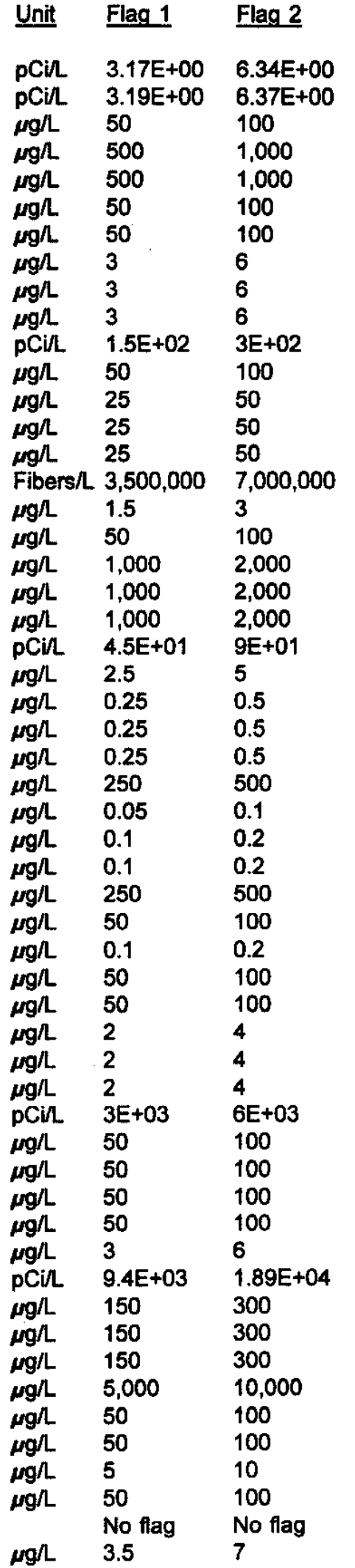

Source

Proposed PDWS (EPA, 1991)

Proposed PDWS (EPA, 1991)

EPA Method 8270

APHA Method 417B

EPA Method 350.1

EPA Method 8270

EPA Method 8270

Final PDWS (EPA, 1993a)

Final PDWS (EPA, 1993a)

Final PDWS (EPA, 1993a)

Interim Final PDWS (EPA, 1977)

EPA Method 8270

Final PDWS (EPA, 1993a)

Final PDWS (EPA, 1993a)

Final PDWS (EPA, 1993a)

Final PDWS (EPA, 1993a)

Final PDWS (EPA, 1993a)

EPA Method 625

Final PDWS (EPA, 1993a)

Final PDWS (EPA, 1993a)

Final PDWS (EPA, 1993a)

Interim Final PDWS (EPA, 1977)

Final PDWS (EPA, 1993a)

EPA Method 8080

EPA Method 8080

EPA Method 8080

EPA Method 8270

Proposed PDWS (EPA, 1990)

Proposed PDWS (EPA, 1990)

Proposed PDWS (EPA, 1990)

EPA Method 8270

EPA Method 8270

Final PDWS (EPA, 1993a)

EPA Method 8270

EPA Method 8270

Final PDWS (EPA, 1993a)

Final PDWS (EPA, 1993a)

Final PDWS (EPA, 1993a)

Interim Final PDWS (EPA, 1977)

EPA Method 8270

EPA Method 8270

EPA Method 8270

EPA Method 8270

Final PDWS (EPA, 1993a)

Proposed PDWS (EPA, 1991)

EPA Method 6010

EPA'Method 6010

EPA Method 6010

EPA Method 300.0

Final PDWS (EPA, 1993a)

Final PDWS (EPA, 1993a)

EPA Method 8240

EPA Method 8270

Set by EPD/EMS

Final PDWS (EPA, 1993a) 
Analyte

Cadmium

Cadmium, dissolved

Cadmium, total recoverable

Calcium

Calcium, dissolved

Calcium, total recoverable

Carbofuran

Carbon-14

Carbonate

Carbon disulfide

Carbon tetrachloride

Cerium-141 ${ }^{\mathrm{C}}$

Cerium-144

Cesium-134

Cesium-137

Chlordane

Chloride

4-Chloroaniline

Chlorobenzene

Chlorobenzilate

4-Chloro-m-cresol

Chloroethane

Chloroethene (Vinyl chloride)

Chloroethyl vinyl ether

2-Chloroethyl vinyl ether

Chloroform

Chloromethane (Methyl chloride)

2-Chloronaphthalene

2-Chlorophenol

4-Chlorophenyl phenyl ether

Chloroprene

Chromium

Chromium, dissolved

Chromium, total recoverable

Chromium-51 ${ }^{\mathrm{C}}$

Chrysene

Cobalt

Cobalt, dissolved

Cobalt, total recoverable

Cobalt-57

Cobalt-58

Cobalt-60

Color

Copper

Copper, dissolved

Copper, total recoverable

Corrosivity

m-Cresol (3-Methyiphenol)

o-Cresol (2-Methylphenol)

p-Cresol (4-Methylphenol)

Curium-242

Curium-243

Curium-243/244

Curium-244

Curium-245/246 ${ }^{\circ}$

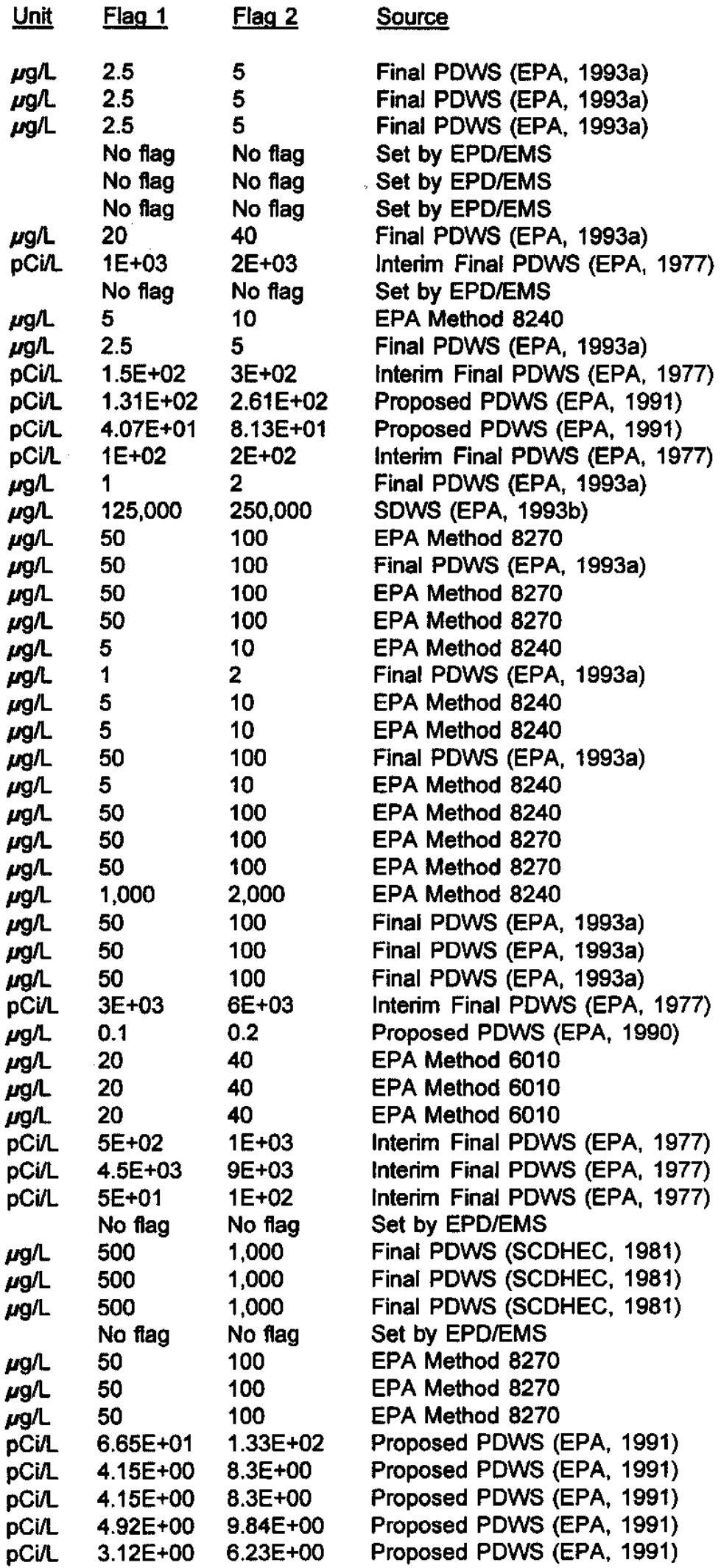




\begin{tabular}{|c|c|c|c|c|}
\hline Analyte & $\underline{\text { Unit }}$ & Flag 1 & Flag 2 & $\underline{\text { Source }}$ \\
\hline $\begin{array}{l}\text { Curium-246 } \\
\text { Cyanide } \\
\text { Dalapon }\end{array}$ & $\begin{array}{l}\mathrm{pCi} \Omega \\
\mu g / L\end{array}$ & $\begin{array}{l}3.14 E+00 \\
100 \\
100\end{array}$ & $\begin{array}{l}6.27 E+00 \\
200 \\
200\end{array}$ & $\begin{array}{l}\text { Proposed PDWS (EPA, 1991) } \\
\text { Final PDWS (EPA, 1993a) } \\
\text { Final PDWS (EPA, 1993a) }\end{array}$ \\
\hline$p, p^{\prime}-D D D$ & $\mu g / L$ & 0.5 & 1 & $\begin{array}{l}\text { Final PDWS (EPA, 1993a) } \\
\text { EPA Method } 8080\end{array}$ \\
\hline$p, p^{\prime}-D D E$ & $\mu g / 2$ & 0.5 & 1 & EPA Method 8080 \\
\hline p,p'-DDT & $\mu g / h$ & 0.5 & 1 & EPA Method 8080 \\
\hline Diallate & $\mu g / \Lambda$ & 50 & 100 & EPA Method 8270 \\
\hline Dibenz[a,h]anthracene & $\mu \mathrm{g} / \mathrm{h}$ & 0.15 & 0.3 & Proposed PDWS (EPA, 1990) \\
\hline Dibenzofuran & $\mu g / L$ & 50 & 100 & EPA Method 8270 \\
\hline Dibromochloromethane & $\mu g / L$ & 50 & 100 & Final PDWS (EPA, 1993a) \\
\hline 1,2-Dibromo-3-chloropropane & $\mu \mathrm{gh}$ & 0.1 & 0.2 & Final PDWS (EPA, 1993a) \\
\hline 1,2-Dibromoethane & $\mu g / L$ & 0.025 & 0.05 & Final PDWS (EPA, 1993a) \\
\hline $\begin{array}{l}\text { Dibromomethane } \\
\text { (Methylene bromide) }\end{array}$ & $\mu g / 2$ & 5 & 10 & EPA Method 8240 \\
\hline Di-n-butyl phthalate & & No flag & No flag & Set by EPD/EMS \\
\hline 1,2-Dichlorobenzene & $\mu g / 2$ & & & Final PDWS (EPA, 1993a) \\
\hline 1,3-Dichlorobenzene & $\mu g / L$ & & 100 & EPA Method 8270 \\
\hline 1,4-Dichlorobenzene & $\mu g / L$ & 37.5 & 75 & Final PDWS (EPA, 1993a) \\
\hline 3,3'-Dichlorobenzidine & $\mu g / L$ & 50 & 100 & EPA Method 8270 \\
\hline trans-1,4-Dichloro-2-butene & $\mu g / L$ & 150 & 300 & EPA Method 8240 \\
\hline Dichlorodifluoromethane & $\mu g / L$ & 5 & 10 & EPA Method 8240 \\
\hline 1,1-Dichloroethane & $\mu g \Lambda$ & 5 & 10 & EPA Method 8240 \\
\hline 1,2-Dichloroethane & $\mu g /$ & 2.5 & 5 & Final PDWS (EPA, 1993a) \\
\hline 1,1-Dichloroethylene & $\mu g / L$ & 3.5 & 7 & Final PDWS (EPA, 1993a) \\
\hline 1,2-Dichloroethylene & $\mu g / L$ & 25 & 50 & Final PDWS (EPA, 1993a) \\
\hline cis-1,2-Dichloroethylene & $\mu g h$ & 35 & 70 & Final PDWS (EPA, 1993a) \\
\hline trans-1,2-Dichloroethylene & $\mu \mathrm{g} / \mathrm{L}$ & 50 & 100 & Final PDWS (EPA, 1993a) \\
\hline $\begin{array}{l}\text { Dichloromethane } \\
\text { (Methylene chloride) }\end{array}$ & $\mu g h$ & 2.5 & 5 & Final PDWS (EPA, 1993a) \\
\hline 2,4-Dichlorophenol & $\mu g / L$ & 50 & 100 & EPA Method 8270 \\
\hline 2,6-Dichlorophenol & $\mu g / L$ & 50 & 100 & EPA Method 8270 \\
\hline 2,4-Dichlorophenoxyacetic acid & $\mu g / L$ & 35 & 70 & Final PDWS (EPA, 1993a) \\
\hline 1,2-Dichloropropane & $\mu g / L$ & 2.5 & 5 & Final PDWS (EPA, 1993a) \\
\hline cis-1,3-Dichloropropene & $\mu g / L$ & 5 & 10 & EPA Method 8240 \\
\hline trans-1,3-Dichloropropene & $\mu \mathrm{g} / \mathrm{L}$ & 5 & 10 & EPA Method 8240 \\
\hline Dieldrin & $\mu g / h$ & 2.5 & 5 & EPA Method 8080 \\
\hline Di(2-ethylhexyl) adipate & $\mu g / L$ & 200 & 400 & Final PDWS (EPA, 1993a) \\
\hline Diethyl phthalate & & No flag & No flag & Set by EPD/EMS \\
\hline Dimethoate & $\mu \mathrm{gg} / \mathrm{L}$ & & 100 & EPA Method 8270 \\
\hline p-Dimethylaminoazobenzene & $\mu g / L$ & 50 & 100 & EPA Method 8270 \\
\hline p-(Dimethylamino)ethylbenzene & $\mu g / L$ & 50 & 100 & EPA Method 8270 \\
\hline 7,12-Dimethylbenz[a]anthracene & $\mu \mathrm{g} / \mathrm{L}$ & 50 & 100 & EPA Method 8270 \\
\hline 3,3'-Dimethylbenzidine & $\mu g / L$ & 50 & 100 & EPA Method 8270 \\
\hline a,a-Dimethylphenethylamine & $\mu \mathrm{g} / \mathrm{L}$ & 50 & 100 & EPA Method 8270 \\
\hline 2,4-Dimethyl phenol & $\mu g h$ & & 100 & EPA Method 8270 \\
\hline Dimethyl phthalate & & No flag & No flag & Set by EPD/EMS \\
\hline 1,3-Dinitrobenzene & $\mu g / L$ & & 100 & EPA Method 8270 \\
\hline 2,4-Dinitrophenol & $\mu g / L$ & 250 & 500 & EPA Method 8270 \\
\hline 2,4-Dinitrotoluene & $\mu \mathrm{g} / \mathrm{L}$ & 50 & 100 & EPA Method 8270 \\
\hline 2,6-Dinitrotoluene & $\mu g / L$ & 50 & 100 & EPA Method 8270 \\
\hline Di-n-octyl phthalate & & No flag & No flag & Set by EPD/EMS \\
\hline 1,4-Dioxane & $\mu g / L$ & 50 & 100 & EPA Method 8270 \\
\hline Diphenylamine & $\mu g / L$ & 50 & 100 & EPA Method 8270 \\
\hline 1,2-Diphenylhydrazine & $\mu g / L$ & $\begin{array}{l}50 \\
10\end{array}$ & 100 & EPA Method 8270 \\
\hline & $\mu g \Omega$ & 10 & 20 & Final PDWS (EPA, 1993a) \\
\hline
\end{tabular}


Analyte

Dissolved organic carbon

Disulfoton

Eh

Endosulfan I

Endosulfan II

Endosulfan sulfate

Endothall ${ }^{b}$

Endrin

Endrin aldehyde

Endrin ketone

Ethylbenzene

Ethyl methacrylate

Ethyl methanesulfonate

Europium-152

Europium-154

Europium-155

Famphur

Fluoranthene

Fluorene

Fluoride

Glyphosate $^{\text {b }}$

Gross alpha

Heptachlor

Heptachlor epoxide

Heptachlorodibenzo-p-dioxin isomers

1,2,3,4,6,7,8-HPCDD

Heptachlorodibenzo-p-furan isomers

1,2,3,4,6,7,8-HPCDF

Hexachlorobenzene

Hexachlorobutadiene

Hexachlorocyclopentadiene

Hexachlorodibenzo-p-dioxin

isomers

1,2,3,4,7,8-HXCDD

Hexachlorodibenzo-p-furan isomers

$1,2,3,4,7,8-H X C D F$

Hexachloroethane

Hexachlorophene

Hexachloropropene

2-Hexanone

Indeno[1,2,3-c, d]pyrene

lodine

lodine-129

lodine-131 ${ }^{\mathrm{c}}$

lodomethane (Methyl iodide)

Iron

Iron, dissolved

Iron, total recoverable

Iron- $55^{c}$

Iron-59

Isobutyl alcohol

Isodrin

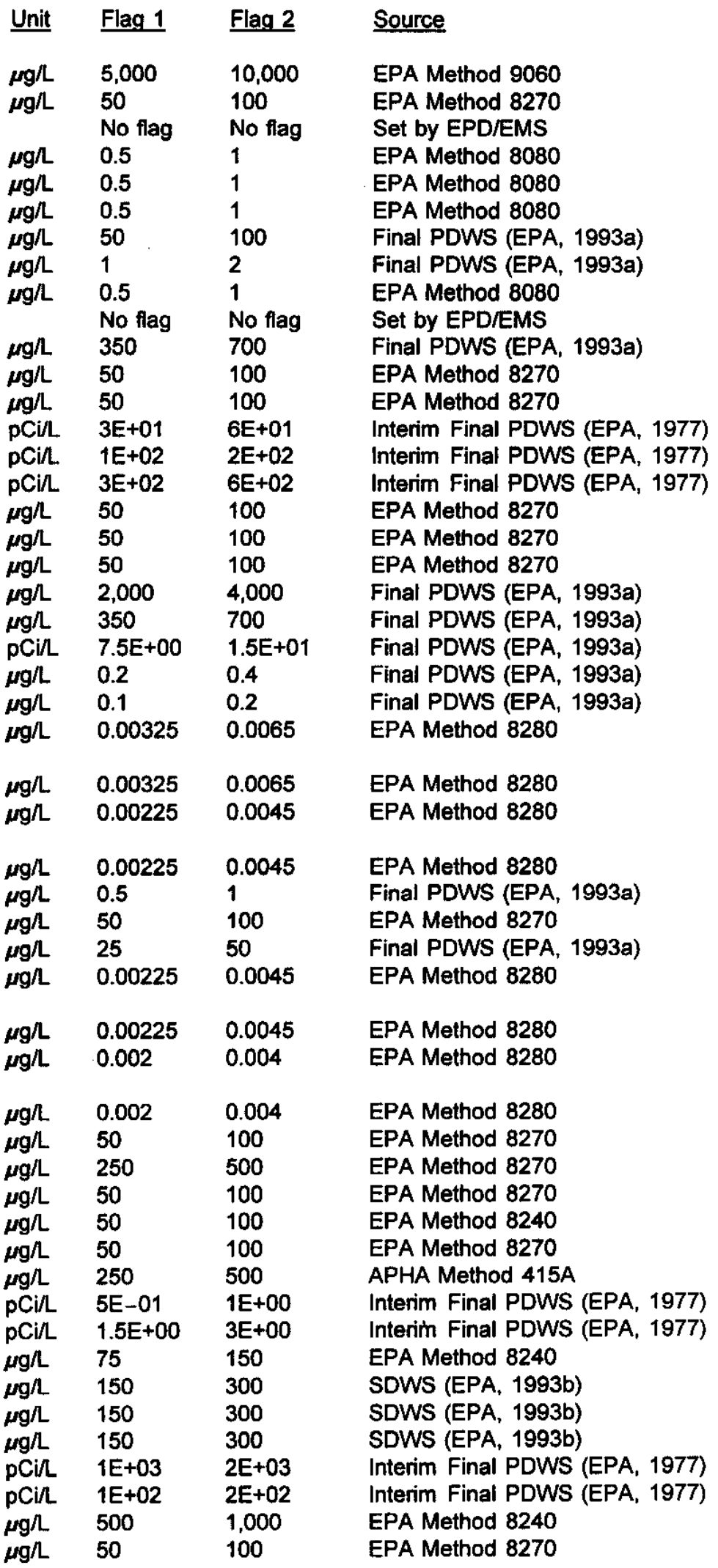


Analvte

Isophorone

Isosafrole

Kepone

Lanthanum-140

Lead

Lead, dissolved

Lead, total recoverable

Lead-212

Lindane

Lithium

Lithium, dissolved

Lithium, total recoverable

Magnesium

Magnesium, dissolved

Magnesium, total recoverable

Manganese

Manganese, dissolved

Manganese, total recoverable

Manganese-54

Mercury

Mercury, dissolved

Mercury, total recoverable

Methacrylonitrile

Methapyrilene

Methoxychlor

3-Methylcholanthrene

2-Methyl-4,6-dinitrophenol

Methyl ethyl ketone

Methyl isobutyl ketone

Methyl methacrylate

Methyl methanesulfonate

2-Methylnaphthalene

Molybdenum

Molybdenum, dissolved

Molybdenum, total recoverable

Naphthalene

1,4-Naphthoquinone

1-Naphthylamine

2-Naphthylamine

Neptunium-237

Nickel

Nickel, dissolved

Nickel, total recoverable

Nickel-59 ${ }^{\mathrm{c}}$

Nickel-63

Niobium-95c

Nitrate as nitrogen

Nitrate-nitrite as nitrogen

Nitrite as nitrogen

m-Nitroaniline

o-Nitroaniline

p-Nitroaniline

Nitrobenzene

Nitrogen by Kjeldahl method

2-Nitrophenol
Unit Flag 1 Flag 2 Source

$\mu \mathrm{g} / \mathrm{50} \quad 100$

$\mu g / 2 \quad 50 \quad 100$

$\mu g / L \quad 50 \quad 100$

$\mathrm{pCi} / \mathrm{L} \quad 3 \mathrm{E}+01 \quad 6 \mathrm{E}+01$

$\mu \mathrm{g} / \mathrm{L} \quad 25 \quad 50$

$\mu g / L \quad 25 \quad 50$

$\mu g / L 25 \quad 50$

pCin 6.2E+01 1.23E+02

$\begin{array}{lll}\mu g / L & 0.1 & 0.2\end{array}$

$\mu g / L \quad 25 \quad 50$

$\mu g / 2 \quad 25 \quad 50$

$\mu \mathrm{gh} 25 \quad 50$

No flag No flag

No flag No flag

No flag No fiag

$\mu \mathrm{g} / \mathrm{L} \quad 25 \quad 50$

$\mu \mathrm{g} / \mathrm{L} \quad 25 \quad 50$

$\mu \mathrm{g} / \mathrm{L} \quad 25 \quad 50$

pCil $1.5 E+02 \quad 3 E+02$

$\mu g / 2112$

$\mu g / 2 \quad 1 \quad 2$

$\mu g / L \quad 1 \quad 2$

$\mu g / L \quad 250 \quad 500$

$\mu \mathrm{g} / \mathrm{L} \quad 50 \quad 100$

$\mu g / 2040$

$\mu g / \mathrm{h} \quad 50 \quad 100$

$\mu g / L \quad 250 \quad 500$

No flag No flag

No flag No flag

$\mu g / L \quad 50 \quad 100$

$\mu$ gl $50 \quad 100$

$\mu g$ h $\quad 50 \quad 100$

$\mu \mathrm{g} / \mathrm{L} \quad 250 \quad 500$

$\mu \mathrm{g} / \mathrm{h} \quad 250 \quad 500$

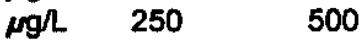

$\mu \mathrm{g} / \mathrm{L} \quad 50 \quad 100$

$\mu g / L \quad 50 \quad 100$

$\mu g / 2 \quad 50 \quad 100$

$\mu g / L \quad 50 \quad 100$

$\mathrm{pCi} / 2.53 \mathrm{E}+00 \quad 7.06 \mathrm{E}+00$

$\mu \mathrm{g} / \mathrm{L} \quad 50 \quad 100$

$\mu g / L \quad 50 \quad 100$

$\mu g / L \quad 50 \quad 100$

PCil 1.5E $+02 \quad 3 E+02$

PCir 2.5E+01 5E+01

pCin 1.5E+02 3.E+02

$\mu \mathrm{g} / \mathrm{L} \quad 5,000 \quad 10,000$

wgh $\quad 5,000 \quad 10,000$

$\mathrm{\mu gh} \quad 500 \quad 1,000$

$\mathrm{mgh} \quad 50 \quad 100$

$\mu g / L \quad 50 \quad 100$

$\mu \mathrm{g} / \mathrm{L} \quad 50 \quad 100$

$\mu g / L \quad 50 \quad 100$

$\mu g / L \quad 500 \quad 1,000$

$\mu g / L \quad 50 \quad 100$
EPA Method 8270

EPA Method 8270

EPA Method 8270

Interim Final PDWS (EPA, 1977)

Final PDWS (SCDHEC, 1981)

Final PDWS (SCDHEC, 1981)

Final PDWS (SCDHEC, 1981)

Proposed PDWS (EPA, 1991)

Final PDWS (EPA, 1993a)

EPA Method 6010

EPA Method 6010

EPA Method 6010

Set by EPD/EMS

Set by EPD/EMS

Set by EPD/EMS

SDWS (EPA, 1993b)

SDWS (EPA, 1993b)

SDWS (EPA, 1993b)

Interim Final PDWS (EPA, 1977)

Final PDWS (EPA, 1993a)

Final PDWS (EPA, 1993a)

Final PDWS (EPA, 1993a)

EPA Method 8240

EPA Method 8270

Final PDWS (EPA, 1993a)

EPA Method 8270

EPA Method 8270

Set by EPD/EMS

Set by EPD/EMS

EPA Method 8270

EPA Method 8270

EPA Method 8270

EPA Method 6010

EPA Method 6010

EPA Method 6010

EPA Method 8270

EPA Method 8270

EPA Method 8270

EPA Method 8270

Proposed PDWS (EPA, 1991)

Final PDWS (EPA, 1993a)

Final PDWS (EPA, 1993a)

Final PDWS (EPA, 1993a)

Interim Final PDWS (EPA, 1977)

Interim Final PDWS (EPA, 1977)

Interim Final PDWS (EPA, 1977)

Final'PDWS (EPA, 1993a)

Final PDWS (EPA, 1993a)

Final PDWS (EPA, 1993a)

EPA Method $\mathbf{8 2 7 0}$

EPA Method 8270

EPA Method 8270

EPA Method 8270

EPA Method 351.2

EPA Method 8270 
Analyte

4-Nitrophenol

4-Nitroquinoline-1-oxide

N-Nitrosodi-n-butylamine

$\mathrm{N}$-Nitrosodiethylamine

N-Nitrosodimethylamine

$\mathrm{N}$-Nitrosodiphenylamine

$\mathrm{N}$-Nitrosodipropylamine

$\mathrm{N}$-Nitrosomethylethylamine

$\mathrm{N}$-Nitrosomorpholine

$\mathrm{N}$-Nitrosopiperidine

$\mathrm{N}$-Nitrosopyrrolidine

5-Nitro-o-toluidine

Nonvolatile beta

Octachlorodibenzo-p-dioxin isomers

Octachlorodibenzo-p-furan isomers

Odor

Oil \& Grease

Oxamyl

Parathion

Parathion methyl

PCB 1016

PCB 1221

PCB 1232

PCB 1242

PCB 1248

PCB 1254

PCB 1260

PCB 1262

Pentachlorobenzene

Pentachlorodibenzo-p-dioxin isomers

1,2,3,7,8-PCDD

Pentachlorodibenzo-p-furan isomers

1,2,3,7,8-PCDF

Pentachloroethane

Pentachloronitrobenzene

Pentachlorophenol

$\mathrm{pH}$

$\mathrm{pH}$

Phenacetin

Phenanthrene

Phenol

Phenols

p-Phenylenediamine

Phorate

Picloram ${ }^{b}$

2-Picoline

Plutonium-238

Plutonium-239

Plutonium-239/240

Plutonium-240

Plutonium-241 ${ }^{\mathrm{C}}$

\begin{tabular}{|c|c|c|c|}
\hline Unit & Flag 1 & Flag 2 & Source \\
\hline$\mu g / L$ & 50 & 100 & EPA Method 8270 \\
\hline$\mu g / L$ & 50 & 100 & EPA Method 8270 \\
\hline$\mu g / L$ & 50 & 100 & EPA Method 8270 \\
\hline$\mu g / L$ & 50 & 100 & EPA Method 8270 \\
\hline$\mu g / L$ & 50 & 100 & EPA Method 8270 \\
\hline$\mu g / L$ & 50 & 100 & EPA Method 8270 \\
\hline$\mu g / L$ & 50 & 100 & EPA Method 8270 \\
\hline$\mu g /$ & 50 & 100 & EPA Method 8270 \\
\hline$\mu g / L$ & 50 & 100 & EPA Method 8270 \\
\hline$\mu g /$ & 50 & 100 & EPA Method 8270 \\
\hline$\mu g / L$ & 50 & 100 & EPA Method 8270 \\
\hline$\mu g / L$ & 50 & 100 & EPA Method 8270 \\
\hline $\mathrm{pCi} / \mathrm{L}$ & $2.5 E+01$ & $5 E+01$ & Interim Final PDWS (EPA, 1977) \\
\hline$\mu \mathrm{g} / \mathrm{L}$ & 0.005 & 0.01 & EPA Method 8280 \\
\hline$\mu g / L$ & 0.005 & 0.01 & EPA Method 8280 \\
\hline & No flag & No flag & Set by EPD/EMS \\
\hline$\mu g / L$ & 5,000 & 10,000 & EPA Method 413.1 \\
\hline$\mu \mathrm{g} / \mathrm{L}$ & 100 & 200 & Final PDWS (EPA, 1993a) \\
\hline$\mu g / L$ & 0.25 & 0.5 & EPA Method 8080 \\
\hline$\mu g /$ & 0.25 & 0.5 & EPA Method 8080 \\
\hline$\mu g / L$ & 0.25 & 0.5 & Final PDWS (EPA, 1993a) \\
\hline$\mu g / L$ & 0.25 & 0.5 & Final PDWS (EPA, 1993a) \\
\hline$\mu g / L$ & 0.25 & 0.5 & Final PDWS (EPA, 1993a) \\
\hline$\mu g / L$ & 0.25 & 0.5 & Final PDWS (EPA, 1993a) \\
\hline$\mu g / L$ & 0.25 & 0.5 & Final PDWS (EPA, 1993a) \\
\hline$\mu g / L$ & 0.25 & 0.5 & Final PDWS (EPA, 1993a) \\
\hline$\mu g h$ & 0.25 & 0.5 & Final PDWS (EPA, 1993a) \\
\hline$\mu g h$ & 0.25 & 0.5 & Final PDWS (EPA, 1993a) \\
\hline$\mu g / L$ & 50 & 100 & EPA Method 8270 \\
\hline$\mu \mathrm{g} / \mathrm{L}$ & 0.00275 & 0.0055 & EPA Method 8280 \\
\hline$\mu g / L$ & 0.00275 & 0.0055 & EPA Method 8280 \\
\hline$\mu g / L$ & 0.00275 & 0.0055 & EPA Method 8280 \\
\hline$\mu g / L$ & 0.00275 & 0.0055 & EPA Method 8280 \\
\hline$\mu g / L$ & 50 & 100 & EPA Method 8270 \\
\hline$\mu g / L$ & 50 & 100 & EPA Method 8270 \\
\hline$\mu g / L$ & 0.5 & 1 & Final PDWS (EPA, 1993a) \\
\hline $\mathrm{pH}$ & 8 & 10 & Set by EPD/EMS \\
\hline $\mathrm{pH}$ & 4 & 3 & Set by EPD/EMS \\
\hline$\mu \mathrm{g} / \mathrm{L}$ & 50 & 100 & EPA Method 8270 \\
\hline$\mu g /$ & 50 & 100 & EPA Method 8270 \\
\hline$\mu g h$ & 50 & 100 & EPA Method 8270 \\
\hline$\mu g /$ & 25 & 50 & EPA Method 420.1 \\
\hline$\mu g / L$ & 50 & 100 & EPA Method 8270 \\
\hline$\mu \mathrm{g} / \mathrm{L}$ & 0.5 & 1 & EPA Method 8080 \\
\hline$\mu g / h$ & 250 & 500 & Final PDWS (EPA, 1993a) \\
\hline$\mu g / L$ & 50 & 100 & EPA Method 8270 \\
\hline pCil & $3.51 \mathrm{E}+00$ & $7.02 \mathrm{E}+00$ & Proposed PDWS (EPA, 1991) \\
\hline $\mathrm{pCi} \Omega$ & $3.11 E+01$ & $6.21 E+01$ & Proposed PDWS (EPA, 1991) \\
\hline $\mathrm{pCi}$ & $3.11 E+01$ & $6.21 E+01$ & Proposed PDWS (EPA, 1991) \\
\hline $\mathrm{pCi} / \mathrm{L}$ & $3.11 E+01$ & $6.22 \mathrm{E}+01$ & Proposed PDWS (EPA, 1991) \\
\hline $\mathrm{pCi} / \mathrm{L}$ & $3.13 E+01$ & $6.26 E+01$ & Proposed PDWS (EPA, 1991) \\
\hline
\end{tabular}


Analyte

Plutonium-242 ${ }^{\mathrm{C}}$

Potassium

Potassium, dissolved

Potassium, total recoverable

Potassium -40

Promethium-144

Promethium-146

Promethium-147

Pronamid

Propionitrile

Pyrene

Pyridine

Radium (alpha-emitting) ${ }^{f}$

Radium-226

Radium-228

Radon-222

Ruthenium-103 ${ }^{\mathrm{C}}$

Ruthenium-106

Safrole

Selenium

Selenium, dissolved

Selenium, total recoverable

Silica

Silica, dissolved

Silica, total recoverable

Silver

Silver, dissolved

Silver, total recoverable

Simazine

Sodium

Sodium, dissolved

Sodium, total recoverable

Sodium-22

Specific conductance

Strontium-89

Strontium-89/90 ${ }^{\circ}$

Strontium-90

Styrene

Sulfate

Sulfide

Sulfotepp

Surfactants

2,3,7,8-TCDD

$2,3,7,8-\mathrm{TCDF}$

Technetium-99

1,2,4,5-Tetrachlorobenzene

Tetrachlorodibenzo-p-dioxin isomers

Tetrachlorodibenzo-p-furan isomers

1,1,1,2-Tetrachloroethane

1,1,2,2-Tetrachloroethane

Tetrachloroethylene

2,3,4,6-Tetrachlorophenol

Thallium 。

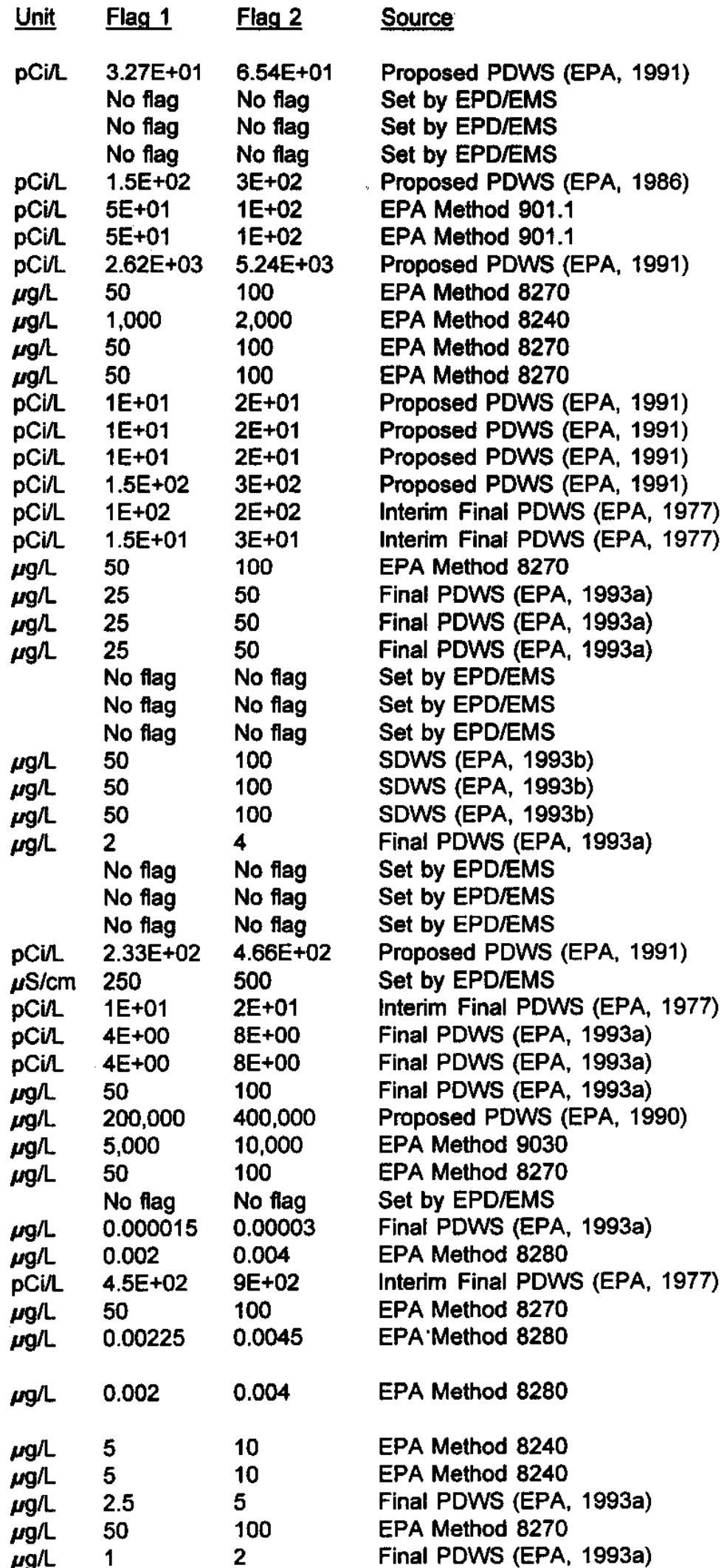




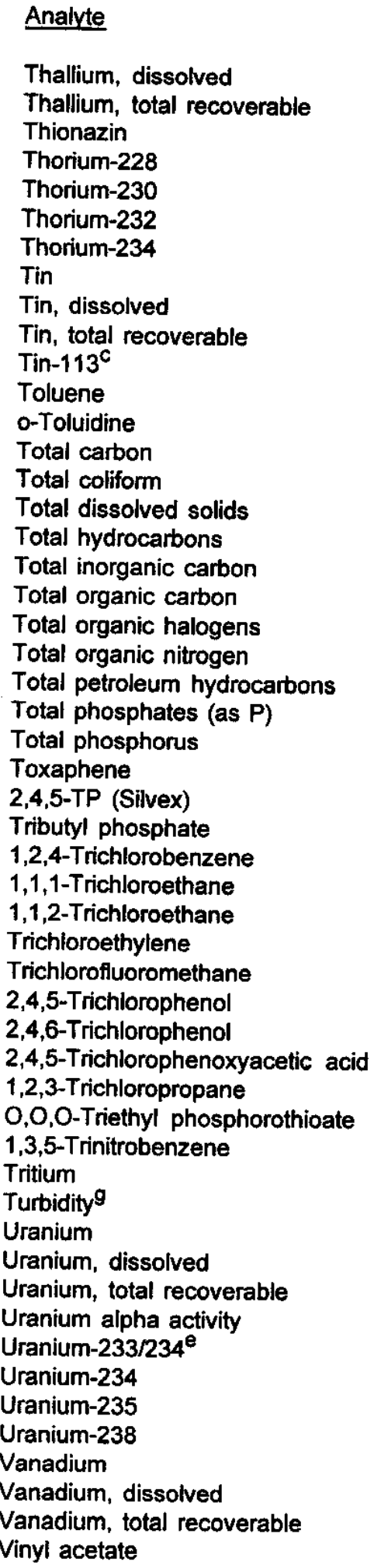

\begin{tabular}{|c|c|c|}
\hline Unit & Flag 1 & Flag 2 \\
\hline$\mu g / L$ & 1 & 2 \\
\hline$\mu g / L$ & 1 & 2 \\
\hline$\mu g / L$ & 50 & 100 \\
\hline $\mathrm{pCi} / \mathrm{L}$ & $6.25 \mathrm{E}+01$ & $1.25 \mathrm{E}+02$ \\
\hline $\mathrm{pCi} / \mathrm{L}$ & $3.96 E+01$ & $7.92 E+01$ \\
\hline $\mathrm{pCi} / \mathrm{L}$ & 4.4E+01 & $8.8 E+01$ \\
\hline pCi/L & $2 \mathrm{E}+02$ & $4.01 E+02$ \\
\hline$\mu g / L$ & 10 & 20 \\
\hline$\mu g / L$ & 10 & 20 \\
\hline$\mu g / L$ & 10 & 20 \\
\hline pCi/l & $1.5 E+02$ & $3 E+02$ \\
\hline$\mu g / L$ & 500 & 1,000 \\
\hline$\mu g /$ & 50 & 100 \\
\hline$\mu g / L$ & $\begin{array}{l}5,000 \\
0\end{array}$ & 10,000 \\
\hline & $\begin{array}{l}0 \\
\text { No flag }\end{array}$ & $\begin{array}{l}0 \\
\text { No flag }\end{array}$ \\
\hline$\mu g / L$ & 5,000 & 10,000 \\
\hline$\mu g / L$ & 5,000 & 10,000 \\
\hline$\mu g / L$ & 5,000 & 10,000 \\
\hline$\mu g / L$ & 25 & 50 \\
\hline$\mu g / L$ & 500 & 1,000 \\
\hline$\mu g / L$ & 5,000 & 10,000 \\
\hline & $\begin{array}{l}\text { No flag } \\
\text { No flag }\end{array}$ & $\begin{array}{l}\text { No flag } \\
\text { No flag }\end{array}$ \\
\hline$\mu g / L$ & 1.5 & 3 \\
\hline$\mu g / L$ & 25 & 50 \\
\hline$\mu g / L$ & 50 & 100 \\
\hline$\mu g /$ & 35 & 70 \\
\hline$\mu g / L$ & 100 & 200 \\
\hline$\mu g / L$ & 2.5 & 5 \\
\hline$\mu g / L$ & 2.5 & 5 \\
\hline$\mu g / L$ & 5 & 10 \\
\hline$\mu g / h$ & 50 & 100 \\
\hline$\mu g / L$ & 50 & 100 \\
\hline$\mu g / L$ & 2.5 & 5 \\
\hline$\mu g / L$ & 5 & 10 \\
\hline$\mu g / L$ & 50 & 100 \\
\hline$\mu g / L$ & 50 & 100 \\
\hline $\mathrm{pCi} / \mathrm{mL}$ & $\begin{array}{l}\text { 1E+01 } \\
\text { No flag }\end{array}$ & $\begin{array}{l}2 E+01 \\
\text { No flag }\end{array}$ \\
\hline$\mu g / L$ & 10 & 20 \\
\hline$\mu g / L$ & 10 & 20 \\
\hline$\mu g / L$ & 10 & 20 \\
\hline $\mathrm{pCi} / \mathrm{L}$ & 1.5E+01 & $3 E+01$ \\
\hline $\mathrm{pCil}$ & $6.9 E+00$ & $1.38 \mathrm{E}+01$ \\
\hline $\mathrm{pCi} / \mathrm{L}$ & $6.95 E+00$ & $1.39 \mathrm{E}+01$ \\
\hline $\mathrm{pCi} / \mathrm{L}$ & $7.25 E+00$ & $1.45 \mathrm{E}+01$ \\
\hline $\mathrm{pCi} / \mathrm{L}$ & $7.3 E+00$ & $1.46 E+01$ \\
\hline$\mu g /$ & 40 & 80 \\
\hline$\mu g / L$ & 40 & 80 \\
\hline$\mu g /$ & 40 & 80 \\
\hline$\mu g / L$ & 5 & 10 \\
\hline
\end{tabular}

\section{Source}

Final PDWS (EPA, 1993a)

Final PDWS (EPA, 1993a)

EPA Method 8270

Proposed PDWS (EPA, 1991)

Proposed PDWS (EPA, 1991)

Proposed PDWS (EPA, 1991)

Proposed PDWS (EPA, 1991)

EPA Method 282.2

EPA Method 282.2

EPA Method 282.2

Interim Final PDWS (EPA, 1977)

Final PDWS (EPA, 1993a)

EPA Method 8270

EPA Method 9060

Final PDWS (EPA, 1993a)

Set by EPD/EMS

EPA Method 418.1

EPA Method 9060

EPA Method 9060

EPA Method 9020

APHA Method 420

EPA Method 418.1

Set by EPD/EMS

Set by EPD/EMS

Final PDWS (EPA, 1993a)

Final PDWS (EPA, 1993a)

EPA Method 8270

Final PDWS (EPA, 1993a)

Final PDWS (EPA, 1993a)

Final PDWS (EPA, 1993a)

Final PDWS (EPA, 1993a)

EPA Method 8240

EPA Method 8270

EPA Method 8270

EPA Method 8150

EPA Method 8240

EPA Method 8270

EPA Method 8270

Final PDWS (EPA, 1993a)

Set by EPD/EMS

Proposed PDWS (EPA, 1991)

Proposed PDWS (EPA, 1991)

Proposed PDWS (EPA, 1991)

Proposed PDWS (EPA, 1991)

Proposed PDWS (EPA, 1991)

Proposed PDWS (EPA, 1991)

Proposed PDWS (EPA, 1991)

Proposed PDWS (EPA, 1991)

EPA Method 6010

EPA Method 6010

EPA Method 6010

EPA Method 8240 


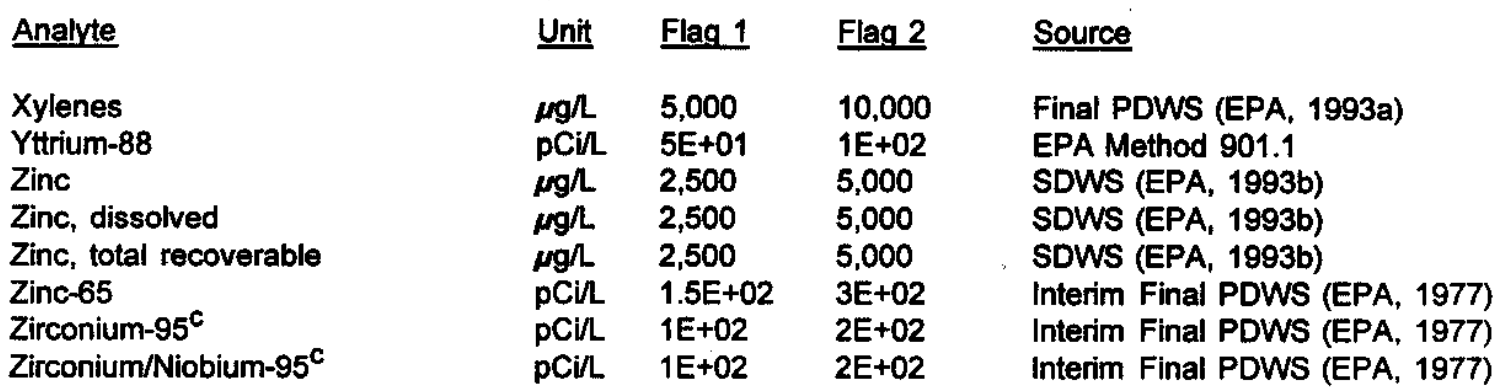

a References for methods are in Appendix E; references for dated sources are at the end of this appendix.

b EMS is currently unable to perform this analysis.

c EMS discontinued monitoring this radionuclide because it is inappropriate for the SRS Groundwater Monitoring Program.

d EPD/EMS set this flagging criterion using the 1991 proposed PDWS because the final PDWS in 1977 may have been in error.

e For double radionuclide analyses where each separate radionuclide has its own standard, the more stringent standard is used.

f The applied standard is for radium-226.

g The primary maximum contaminant level range for turbidity is 1-5 NTU, which is inappropriate for the SRS Groundwater Monitoring Program.

\section{References}

EPA (U.S. Environmental Protection Agency), 1977. National Interim Primary Drinking Water Regulations, EPA-570/9-76-003. Washington, DC.

EPA (U.S. Environmental Protection Agency), 1986. Water Pollution Control; National Primary Drinking Water Regulations, Radionuclides (Proposed). Federal Register, September 30, 1986, pp. 34835-34862. Washington, DC.

EPA (U.S. Environmental Protection Agency), 1990. National Primary and Secondary Drinking Water Regulations; Synthetic Organic Chemicals and Inorganic Chemicals (Proposed Rule).

Federal Register, July 25, 1990, pp. 30369-30448. Washington, DC.

EPA (U.S. Environmental Protection Agency), 1991. National Primary Drinking Water Regulations; Radionuclides; Proposed Rule. Federal Register, July 18, 1991, pp. 33052-33127. Washington, DC.

EPA (U.S. Environmental Protection Agency), 1993a. National Primary Drinking Water Regulations. Code of Federal Regulations, Title 40, Part 141, pp. 592-732. Washington, DC.

EPA (U.S. Environmental Protection Agency), 1993b. National Secondary Drinking Water Regulations. Code of Federal Regulations, Title 40, Part 143, pp. 774-777. Washington, DC.

SCDHEC (South Carolina Department of Health and Environmental Control), 1981. State Primary Drinking Water Regulations, R.61-58.5. Columbia, SC. 
WSRC-TR-94-0491

Unclassified

\section{Appendix C}

Figures 
THIS PAGE LEFT BLANK INTENTIONALLY. 


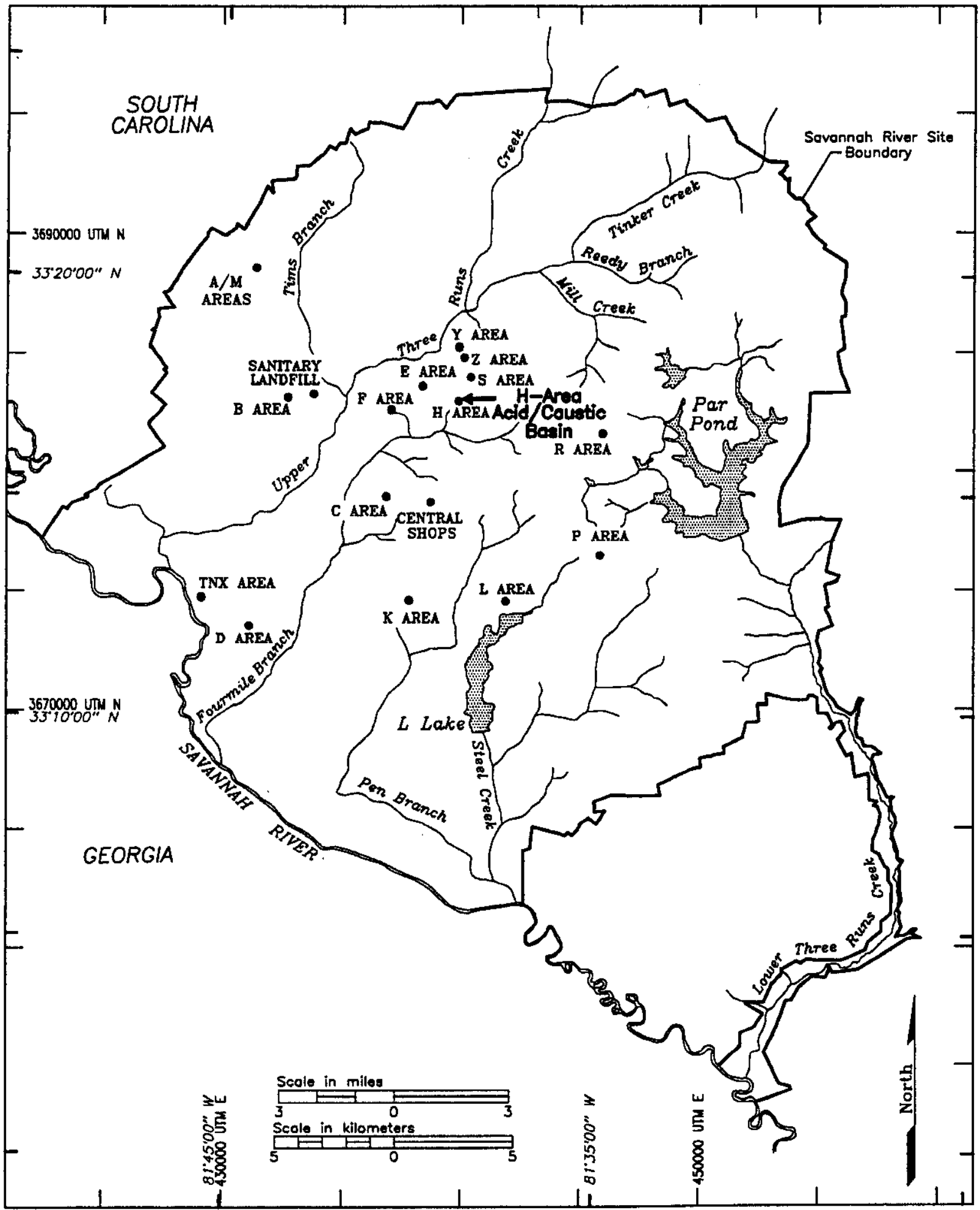

Figure 1. Location of the H-Area Acid/Caustic Basin at the Savannah River Site 


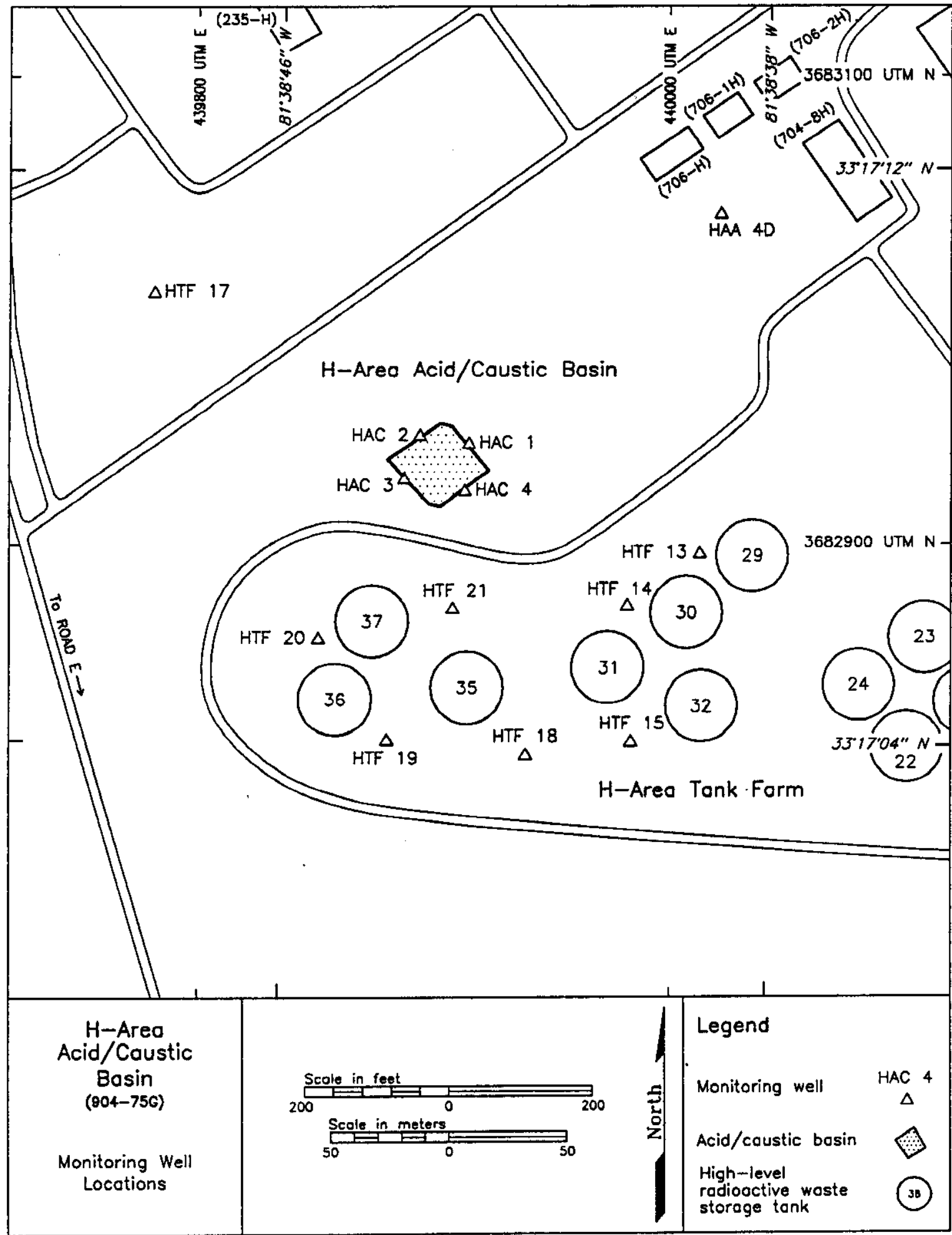

Figure 2. Location of Groundwater Monitoring Wells at the H-Area Acid/Caustic Basin 


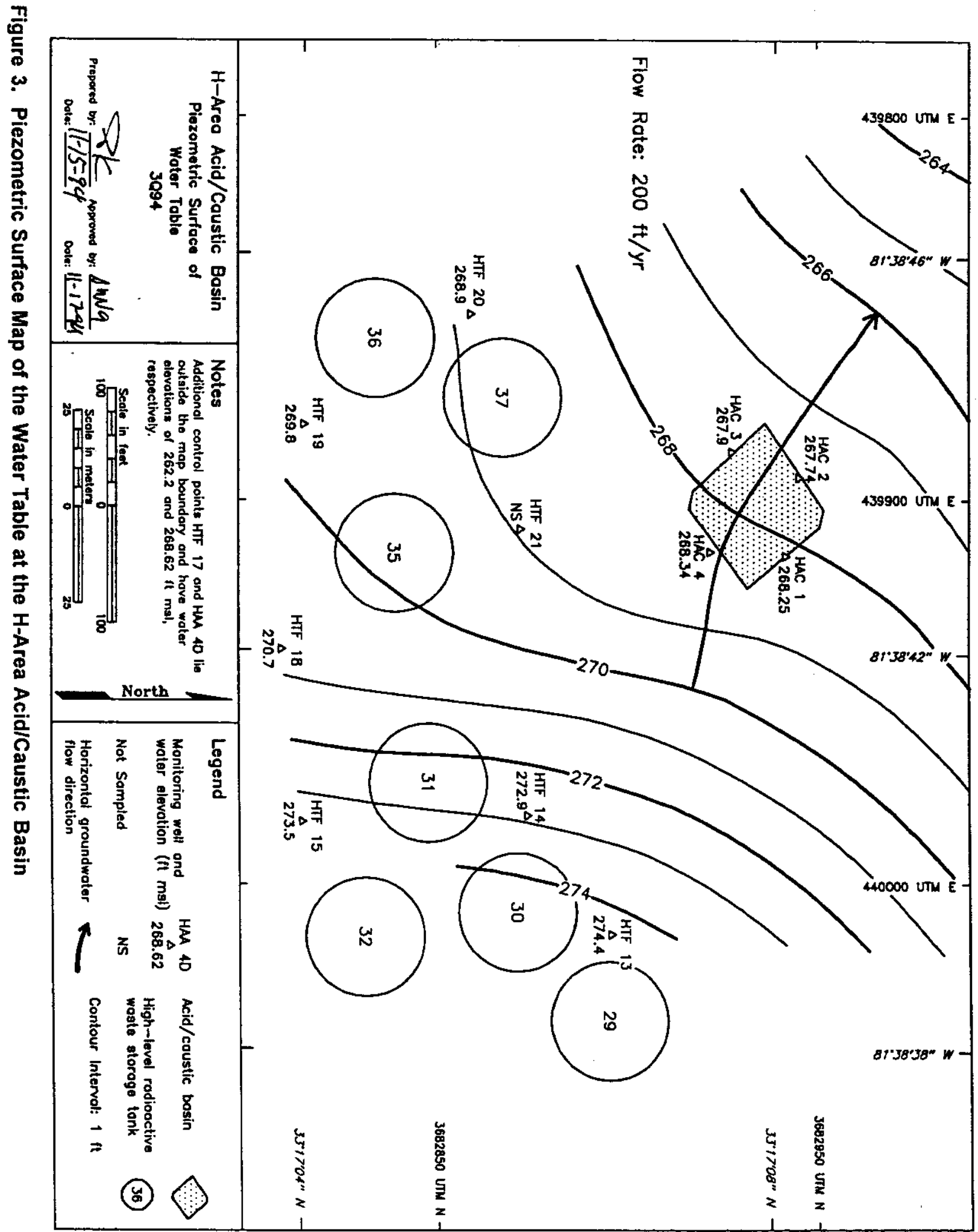


THIS PAGE LEFT BLANK INTENTIONALLY. 
WSRC-TR-94-0491

Unclassified

\section{Appendix D}

\section{Groundwater Monitoring Results Tables}


WSRC-TR-94-0491

Unclassified

THIS PAGE LEFT BLANK INTENTIONALLY. 


\section{Key to Reading the Tables}

The following abbreviations may appear in the data tables:

\section{Constituents}

$1,2,3,4,6,7,8-H P C D D$

$1,2,3,4,6,7,8-\mathrm{HPCDF}$

$1,2,3,4,7,8-\mathrm{HXCDD}$

$1,2,3,4,7,8-\mathrm{HXCDF}$

Lindane

PCB

$1,2,3,7,8-P C D D$

$1,2,3,7,8-P C D F$

Sp. conductance

TCDD

TCDF

\section{Laboratories}

$\mathrm{CN}$

EM

GE and GP

SC

SP

TM

WA and WS

Sampling Codes

B
C
$D$
$E$
I
L
P
S
X

Sampling Methods

B

$P$

$S$

V 1,2,3,4,6,7,8-heptachlorodibenzo-p-dioxin

$1,2,3,4,6,7,8$-heptachlorodibenzo-p-furan

$1,2,3,4,7,8$-hexachlorodibenzo-p-dioxin

1,2,3,4,7,8-hexachlorodibenzo-p-furan

gamma-benzene hexachloride

polychlorinated biphenyl

1,2,3,7,8-pentachlorodibenzo-p-dioxin

1,2,3,7,8-pentachlorodibenzo-p-furan

specific conductance

tetrachlorodibenzo-p-dioxin

tetrachlorodibenzo-p-furan
Clemson Technical Center, Inc.

Environmental Protection Department/Environmental

Monitoring Section (EPD/EMS) Laboratory

General Engineering Laboratories

Savannah River Technology Center

Spencer Testing Services, Inc.

TMAVEberline

Roy F. Weston, Inc.

blank sample was collected

well was pumping continuously

well was dry

equipment blank was collected

well went dry during sampling; insufficient water to collect all samples

well went dry before sampling began; only depth to water can be determined

inaccessibility or mechanical failure prevented sample collection and field analysis of the water no water in standpipe; for water level events only well went dry during purging; samples collected after well recovered

sample collected using an open-bucket bailer sample collected using a bladder pump sample collected using a single-speed centrifugal downhole pump sample collected using a variable-speed pump 
Units

E

$\mathrm{mg} / \mathrm{L}$

$\mathrm{msl}$

MSL

NTU

pCi/L

$\mathrm{pCi} / \mathrm{mL}$

$\mathrm{pH}$

$\mu \mathrm{g} / \mathrm{L}$

$\mu \mathrm{S} / \mathrm{cm}$ exponential notation (e.g., $1.1 \mathrm{E}-09=1.1 \times 10^{-9}=$

0.0000000011 )

milligrams per liter

mean sea level

million structures per liter

turbidity unit

picocuries per liter

picocuries per milliliter

$\mathrm{pH}$ unit

micrograms per liter

microsiemens per centimeter

Other

CS
DF
H
Mod
PDWS
PVC
ST
TOC

carbon steel

dilution factor column in data tables

holding time column in data tables

modifier column in data tables

primary drinking water standard

polyvinyl chloride

exceeded standard column in data tables

top of casing

\section{Holding Times}

Standard analytical methods include a limit, called holding time, on the maximum elapsed time between sample collection and extraction or analysis by the laboratory. In the data tables, a large bullet $(\cdot)$ in the $H$ (holding time) column indicates that holding time was exceeded. Analyses performed beyond holding times may not yield valid results.

The South Carolina Department of Health and Environmental Control allows only 15 minutes to elapse between sampling and analysis for $\mathrm{pH}$. Thus, only field $\mathrm{pH}$ measurements can meet the holding time criterion; laboratory $\mathrm{pH}$ analyses always will exceed it.

The laboratory procedure used for the determination of specific conductance allows one day to elapse between sampling and analysis. Thus, laboratory specific conductance measurements may exceed the holding time criterion.

\section{Data Rounding}

Constituent results in analytical results tables that appear to equal the final PDWS but are not marked in the ST (exceeded the final PDWS or screening level) column are below the final PDWS in the database. Values stored in the database contain more significant digits than the reported results. Apparent discrepancies in the tables are due to the rounding of reported results. 


\section{Data Qualification}

The contract laboratories continually assess their own accuracy and precision according to U.S. Environmental Protection Agency (EPA) guidelines. They submit sample- or batch-specific quality assurance/quality control information either at the same time as analytical results or in a quarterly summary. Properly defined and used result modifiers (also referred to as qualifiers) can be a key component in assessing data usability. Result modifiers designed by the EPD/EMS and provided to the primary laboratories are defined below. These modifiers appear in the data tables under the column Mod. The lettered modifiers are based on EPA's STORET codes.

\section{Result modifier}

Data are not qualified. Numbers should be interpreted exactly as reported.

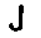

1

L

M

$\mathbf{R}$

T

U

V

Y

1

2

3

4

6
Value is estimated because quantitation in the sample or in associated quality control samples did not meet specifications.

The value in the result field is the instrument reading, not the sample quantification limit. Always used with the result qualifier $U$.

Value is off-scale high. The actual value is not known but is known to be greater than the value shown.

Presence of the analyte is verified but not quantified.

Result was rejected because performance requirements in the sample analysis or associated quality control analyses were not met.

Analyte was not detected; if present, it was below the criteria for detection.

Material analyzed for but not detected. Analytical result reported is less than the sample quantitation limit.

Arialyte was detected in an associated method blank.

Result was obtained from an unpreserved or improperly preserved sample. Data may not be accurate.

Result may be an underestimation of the true value due to analytical bias.

Result may be an overestimation of the true value due to analytical bias.

The associated result may be of poor precision (high variability) due to analytical bias.

Result is associated with QA results indicating matrix interference.

The associated result is from a reanalysis performed out of holding time due to problems with an earlier analysis. 
WSRC-TR-94-0491

Unclassified

Table 1. Maximum Results for Constituents Exceeding Final Primary Drinking Water Standards

\begin{tabular}{|c|c|c|c|c|c|c|}
\hline Well & Constituent & Unit & 4Q93 & 1094 & $\underline{2 Q 94}$ & $\underline{3094}$ \\
\hline HAC 1 & Tritium & $\mathrm{pCi} / \mathrm{mL}$ & $4.6 E+01$ & $6.2 \mathrm{E}+01$ & $6.1 E+01$ & $6.5 E+01$ \\
\hline HAC 2 & Tritium & $\mathrm{pCi} / \mathrm{mL}$ & $3.8 E+01$ & $3.6 \mathrm{E}+01$ & $3.5 E+01$ & $3.9 E+01$ \\
\hline HAC 3 & Tritium & $\mathrm{pCi} / \mathrm{mL}$ & $3.9 E+01$ & $3.6 \mathrm{E}+01$ & $3.6 \mathrm{E}+01$ & 3.7E+01 \\
\hline HAC 4 & $\begin{array}{l}\text { Carbon tetrachloride } \\
\text { Heptachlor epoxide } \\
\text { Tritium }\end{array}$ & $\begin{array}{l}\mu g / 2 \\
\mu g / L \\
p C i / m L\end{array}$ & $\begin{array}{l}N^{a} \\
\text { NA } \\
3.9 E+01\end{array}$ & $\begin{array}{l}7.4 \\
0.22 \\
3.6 \mathrm{E}+01\end{array}$ & $\begin{array}{l}5.1 \\
-b_{b} \\
3.0 E+01\end{array}$ & $\begin{array}{l}8.5 \\
\text { NA } \\
3.3 E+01\end{array}$ \\
\hline
\end{tabular}

Note: The modifier column applies to third quarter 1994 only.

a $N A=$ not analyzed.

b $-=$ not above final PDWS.

Table 2. Maximum Results for Constituents Exceeding Other Flag 2 Criteria or the SRS Turbidity Standard

\begin{tabular}{|c|c|c|c|c|}
\hline Well & Constituent & Unit & 3Q994 & Mod \\
\hline HAC 1 & $\begin{array}{l}\text { Aluminum } \\
\text { Iron }\end{array}$ & $\begin{array}{l}\mu \mathrm{g} / \mathrm{L} \\
\mu \mathrm{g} / \mathrm{L}\end{array}$ & $\begin{array}{l}64 \\
595\end{array}$ & $\begin{array}{l}Y \\
Y\end{array}$ \\
\hline HAC 2 & $\begin{array}{l}\text { Aluminum } \\
\text { Iron } \\
\text { Total organic halogens }\end{array}$ & $\begin{array}{l}\mu g / h \\
\mu g / L \\
\mu g / L\end{array}$ & $\begin{array}{l}162 \\
1,280 \\
110\end{array}$ & $\begin{array}{l}Y \\
Y \\
Y\end{array}$ \\
\hline HAC 3 & $\begin{array}{l}\text { Aluminum } \\
\text { Iron } \\
\text { Manganese }\end{array}$ & $\begin{array}{l}\mu g / L \\
\mu g / L \\
\mu g / L\end{array}$ & $\begin{array}{l}303 \\
678 \\
61\end{array}$ & $\begin{array}{l}Y \\
Y \\
Y\end{array}$ \\
\hline HAC 4 & Aluminum & $\mu g / h$ & 123 & $Y$ \\
\hline
\end{tabular}

Notes: These results do not include field data. The groundwater samples are unfiltered. Thus, the results for metals are for total recoverable metals. Flags are established by EPD/EMS and are based on final PDWS, Secondary Drinking Water Standards, or method detection limits (Appendix B). 
Table 3. Groundwater Monitoring Results for Individual Wells

\section{WELL HAC 1}

\begin{tabular}{|c|c|c|c|c|c|c|}
\hline SRS Coord. & Lat/Longitude & Screen Zone Elevation & Top of Casing & Casing & Pump & Formation \\
\hline $\begin{array}{l}\text { N72171.0 } \\
\text { E61415.2 }\end{array}$ & $\begin{array}{l}33.285599^{\circ} \mathrm{N} \\
81.645272^{\circ} \mathrm{W}\end{array}$ & $278.8-258.8 \mathrm{ft} \mathrm{msl}$ & $298.4 \mathrm{ft} \mathrm{msl}$ & 4" PVC & S & \\
\hline
\end{tabular}

\section{FIELD MEASUREMENTS}

Sample date: 07/20/94

Depth to water: $30.15 \mathrm{ft}(9.19 \mathrm{~m})$ below TOC

Water elevation: $268.25 \mathrm{ft}(81.76 \mathrm{~m}) \mathrm{msl}$

Sp. conductance: $125 \mu \mathrm{S} / \mathrm{cm}$

Turbidity: 0.4 NTU

Water evacuated before sampling: $40 \mathrm{gal}$

\section{LABORATORY ANALYSES}

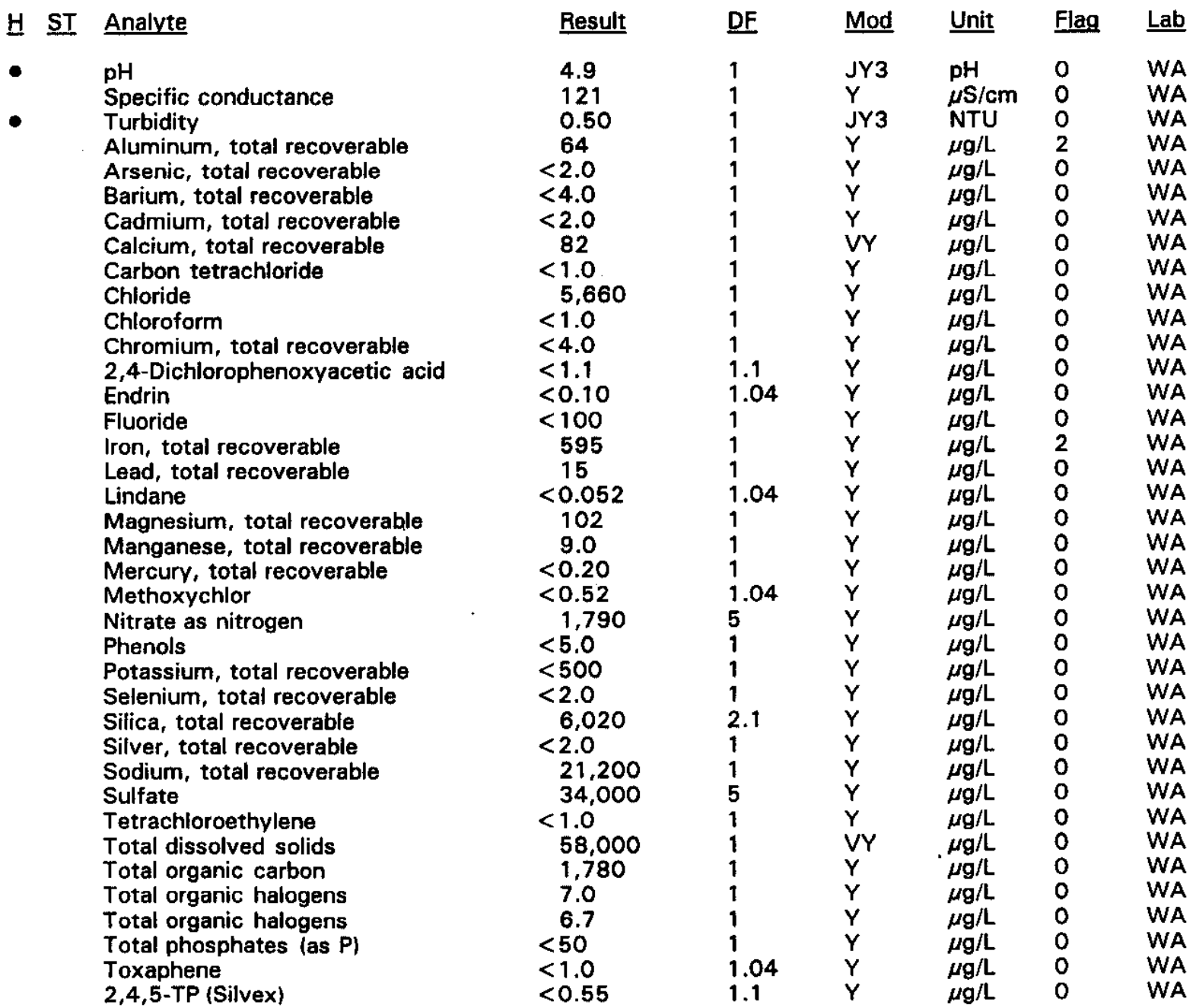

- = exceeded holding time. $\|$ = exceeded screening level or final PDWS.
Time: 13:02

pH: 5.2

Alkalinity: $0 \mathrm{mg} / \mathrm{L}$

Water temperature: $23.6^{\circ} \mathrm{C}$

Volumes purged: 6.5 well volumes 
WELL HAC 1 collected on 07/20/94, laboratory analyses (cont.)

H ST Analyte

1,1,1-Trichloroethane

Trichloroethylene

Gross alpha

Nonvolatile beta

- Tritium

$\begin{aligned} & \text { Result } \\ < & 1.0 \\ < & 1.0 \\ & 2.5 \mathrm{E}-01 \\ & 1.8 \mathrm{E}+00 \\ & 6.5 \mathrm{E}+01\end{aligned}$

$\begin{array}{lllll}\text { Mod } & & \text { Unit } & \text { Flag } & \text { Lab } \\ \text { Y } & & \mu \mathrm{g} / \mathrm{L} & 0 & \text { WA } \\ \mathrm{Y} & \mu \mathrm{g} / \mathrm{L} & 0 & \text { WA } \\ \text { UI } & \mathrm{pCi} / \mathrm{L} & 0 & \text { GP } \\ \mathrm{J} 3 & \mathrm{pCi} / \mathrm{L} & 0 & \text { GP } \\ & \mathrm{pCi} / \mathrm{mL} & 2 & \text { GP }\end{array}$

WELL HAC 2

\begin{tabular}{|c|c|c|c|c|c|c|}
\hline SRS Coord. & Lat/Longitude & Screen Zone Elevation & Top of Casing & Casing & Pump & Formation \\
\hline $\begin{array}{l}\text { N72220.2 } \\
\text { E61366.9 }\end{array}$ & $\begin{array}{l}33.285629^{\circ} \mathrm{N} \\
81.645495^{\circ} \mathrm{W}\end{array}$ & 2 & 29 & 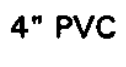 & $s$ & e \\
\hline
\end{tabular}

\section{FIELD MEASUREMENTS}

Sample date: 07/20/94

Depth to water: $30.36 \mathrm{ft}(9.25 \mathrm{~m})$ below TOC

Water elevation: $267.74 \mathrm{ft}(81.61 \mathrm{~m}) \mathrm{msl}$

Sp. conductance: $276 \mu \mathrm{S} / \mathrm{cm}$

Turbidity: 13.7 NTU

Water evacuated before sampling: 4 gal

The well went dry during purging.

\section{LABORATORY ANALYSES}

H $\underline{\text { ST }}$ Analyte

- $\mathrm{pH}$

Specific conductance

Turbidity

Aluminum, total recoverable

Arsenic, total recoverable

Barium, total recoverable

Cadmium, total recoverable

Calcium, total recoverable

Carbon tetrachloride

Chloride

Chloroform

Chromium, total recoverable

2,4-Dichlorophenoxyacetic acid

Endrin

Fluoride

Iron, total recoverable

Lead, total recoverable

Lindane

Magnesium, total recoverable

Manganese, total recoverable

Mercury, total recoverable

Methoxychlor

Nitrate as nitrogen

Phenols

Potassium, total recoverable

Selenium, total recoverable

Silica, total recoverable

Silver, total recoverable

Sodium, total recoverable

Sulfate
Time: 12:22

pH: 5.9

Alkalinity: $28 \mathrm{mg} / \mathrm{L}$

Water temperature: $23.3^{\circ} \mathrm{C}$

Volumes purged: 0.7 well volumes

\begin{tabular}{|c|c|c|c|c|c|}
\hline Result & $\underline{\mathrm{DF}}$ & Mod & $\underline{\text { Unit }}$ & Flag & $\underline{\text { Lab }}$ \\
\hline 5.5 & 1 & JY3 & $\mathrm{pH}$ & 0 & WA \\
\hline$<1.0$ & 1 & $Y$ & $\mu \mathrm{S} / \mathrm{cm}$ & 0 & WA \\
\hline 11 & 1 & JY3 & NTU & 0 & WA \\
\hline 162 & 1 & $Y$ & $\mu \mathrm{g} / \mathrm{L}$ & 2 & WA \\
\hline$<2.0^{-}$ & 1 & $\dot{Y}$ & $\mu \mathrm{g} / \mathrm{L}$ & 0 & WA \\
\hline 5.8 & 1 & $Y$ & $\mu \mathrm{g} / \mathrm{L}$ & 0 & WA \\
\hline$<2.0$ & 1 & $Y$ & $\mu \mathrm{g} / \mathrm{L}$ & 0 & WA \\
\hline 216 & $i$ & VY & $\mu \mathrm{g} / \mathrm{L}$ & 0 & WA \\
\hline$<1.0$ & 1 & $Y$ & $\mu \mathrm{g} / \mathrm{L}$ & 0 & WA \\
\hline 6,250 & $i$ & $\dot{Y}$ & $\mu \mathrm{g} / \mathrm{L}$ & 0 & WA \\
\hline$<1.0$ & 1 & $Y$ & $\mu \mathrm{g} / \mathrm{L}$ & 0 & WA \\
\hline$<4.0$ & 1 & $Y$ & $\mu g / L$ & 0 & WA \\
\hline$<1.1$ & 1.09 & $Y$ & $\mu \mathrm{g} / \mathrm{L}$ & 0 & WA \\
\hline$<0.10$ & 1.04 & $\dot{Y}$ & $\mu \mathrm{g} / \mathrm{L}$ & 0 & WA \\
\hline$<100$ & $i$ & $\dot{Y}$ & $\mu \mathrm{g} / \mathrm{L}$ & 0 & WA \\
\hline 1,280 & 1 & $Y$ & $\mu \mathrm{g} / \mathrm{L}$ & 2 & WA \\
\hline 9.3 & 1 & $Y$ & $\mu \mathrm{g} / \mathrm{L}$ & 0 & WA \\
\hline$<0.052$ & 1.04 & $\dot{Y}$ & $\mu \mathrm{g} / \mathrm{L}$ & 0 & WA \\
\hline 290 & 1 & $Y$ & $\mu \mathrm{g} / \mathrm{L}$ & 0 & WA \\
\hline 20 & 1 & $Y$ & $\mu \mathrm{g} / \mathrm{L}$ & 0 & WA \\
\hline 0.47 & 1 & $Y$ & $\mu \mathrm{g} / \mathrm{L}$ & 0 & WA \\
\hline$<0.52$ & 1.04 & $Y$ & $\mu \mathrm{g} / \mathrm{L}$ & 0 & WA \\
\hline 194 & 1 & $Y$ & $\mu \mathrm{g} / \mathrm{L}$ & 0 & WA \\
\hline$<5.0$ & 1 & $\dot{\gamma}$ & $\mu g / L$ & 0 & WA \\
\hline$<500$ & 1 & $Y$ & $\mu \mathrm{g} / \mathrm{L}$ & 0 & WA \\
\hline$<2.0$ & 1 & $Y$ & $\mu \mathrm{g} / \mathrm{L}$ & 0 & WA \\
\hline 6,070 & 2.1 & $Y$ & $\mu \mathrm{g} / \mathrm{L}$ & 0 & WA \\
\hline$<2.0$ & 1 & $Y$ & $\mu \mathrm{g} / \mathrm{L}$ & 0 & WA \\
\hline 87,700 & 1 & $Y$ & $\mu g / L$ & 0 & WA \\
\hline 164,000 & 50 & $Y$ & $\mu g / L$ & 0 & WA \\
\hline
\end{tabular}

- = exceeded holding time. $=$ exceeded screening level or final PDWS. 
WELL HAC 2 collected on $07 / 20 / 94$, laboratory analyses (cont.)

H $\underline{\text { ST }}$ Analyte

Tetrachloroethylene

Total dissolved solids

Total organic carbon

Total organic halogens

Total phosphates (as P)

Toxaphene

2,4,5-TP (Silvex)

1,1,1-Trichloroethane

Trichloroethylene

Gross alpha

Nonvolatile beta

- Tritium

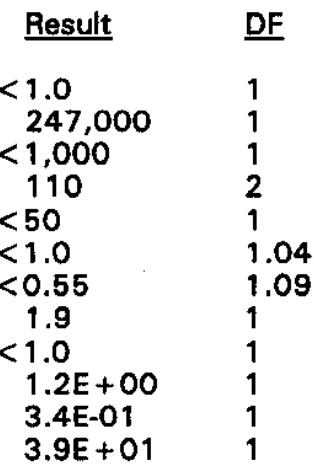

$\begin{array}{lllll}\text { Mod } & & \text { Unit } & \text { Flag } & \text { Lab } \\ Y & & & & \\ Y & & \mu g / L & 0 & \text { WA } \\ V Y & \mu g / L & 0 & \text { WA } \\ Y & \mu g / L & 0 & \text { WA } \\ Y & \mu g / L & 2 & \text { WA } \\ Y & \mu g / L & 0 & \text { WA } \\ Y & \mu g / L & 0 & \text { WA } \\ Y & \mu g / L & 0 & \text { WA } \\ Y & \mu g / L & 0 & \text { WA } \\ Y & \mu g / L & 0 & \text { WA } \\ U I & \mathrm{pCi} / \mathrm{L} & 0 & \text { GP } \\ \text { UI } & \mathrm{pCi} / \mathrm{L} & 0 & \text { GP } \\ & \mathrm{pCi} / \mathrm{mL} & 2 & \text { GP }\end{array}$

\section{WELL HAC 3}

\begin{tabular}{|c|c|c|c|c|c|c|}
\hline SRS Coord. & Lat/Longitude & Screen Zone Elevation & Top of Casing & Casing & Pump & Formation \\
\hline $\begin{array}{l}\text { N72183.4 } \\
\text { E61313.6 }\end{array}$ & $\begin{array}{l}33.285461^{\circ} \mathrm{N} \\
81.645564^{\circ} \mathrm{W}\end{array}$ & $275.0-255.0 \mathrm{ft} \mathrm{msl}$ & $298 \mathrm{ft} \mathrm{msl}$ & 4" PVC & $\mathbf{S}$ & Water Table \\
\hline
\end{tabular}

\section{FIELD MEASUREMENTS}

Sample date: $07 / 21 / 94$

Depth to water: $30.10 \mathrm{ft}(9.17 \mathrm{~m})$ below TOC

Water elevation: $267.90 \mathrm{ft}(81.66 \mathrm{~m}) \mathrm{msl}$

Sp. conductance: $292 \mu \mathrm{S} / \mathrm{cm}$

Turbidity: 39.2 NTU

Water evacuated before sampling: $6 \mathrm{gal}$

The well went dry during purging.

\section{LABORATORY ANALYSES}$$
\text { : }
$$

H ST Analyte

- $\quad \mathrm{pH}$

Specific conductance

Specific conductance

- Turbidity

Turbidity

Aluminum, total recoverable

Arsenic, total recoverable

Barium, total recoverable

Cadmium, total recoverable

Calcium, total recoverable

Carbon tetrachloride

Chloride

Chloroform

Chromium, total recoverable

2,4-Dichlorophenoxyacetic acid

Endrin

Fluoride

Fluoride

Iron, total recoverable

Lead, total recoverable

Lindane

Magnesium, total recoverable

\begin{tabular}{l}
$\quad$ Result \\
\hline 4.9 \\
169 \\
168 \\
3.8 \\
3.5 \\
303 \\
$<2.0$ \\
9.8 \\
$<2.0$ \\
282 \\
$<1.0$ \\
6,800 \\
$<1.0$ \\
$<4.0$ \\
$<1.1$ \\
$<0.10$ \\
$<100$ \\
$<100$ \\
678 \\
12 \\
$<0.052$ \\
280
\end{tabular}

Time: 13:37

pH: 5.1

Alkalinity: $0 \mathrm{mg} / \mathrm{L}$

Water temperature: $23.7^{\circ} \mathrm{C}$

Volumes purged: 0.7 well volumes 
WELL HAC 3 collected on 07/21/94, laboratory analyses (cont.)

H ST Analyte

Manganese, total recoverable

Mercury, total recoverable

Methoxychlor

Nitrate as nitrogen

Phenols

Potassium, total recoverable

Selenium, total recoverable

Silica, total recoverable

Silver, total recoverable

Sodium, total recoverable

Sulfate

Tetrachloroethylene

Total dissolved solids

Total organic carbon

Total organic halogens

Total phosphates (as P)

Toxaphene

2,4,5-TP (Silvex)

$1,1,1$-Trichloroethane

Trichloroethylene

Gross alpha

Nonvolatile beta

a Tritium

$\begin{array}{ll}\text { Result } & \text { DF } \\ 61 & 1 \\ 0.50 & 1 \\ <0.52 & 1.04 \\ 1,600 & 5 \\ <5.0 & 1 \\ <500 & 1 \\ <2.0 & 1 \\ 4,980 & 2.1 \\ <2.0 & 1 \\ 25,100 & 1 \\ 50,400 & 20 \\ <1.0 & 1 \\ 47,000 & 1 \\ <1,000 & 1 \\ 46 & 2 \\ <50 & 1 \\ <1.0 & 1.04 \\ <0.55 & 1.1 \\ 1.7 & 1 \\ <1.0 & 1 \\ 8.8 E-01 & 1 \\ 1.2 E+00 & 1 \\ 3.7 E+01 & 1\end{array}$

\begin{tabular}{|c|c|c|}
\hline Mod & Unit & Flag \\
\hline$Y$ & $\mu \mathrm{g} / \mathrm{L}$ & 2 \\
\hline$Y$ & $\mu \mathrm{g} / \mathrm{L}$ & 0 \\
\hline$Y$ & $\mu \mathrm{g} / \mathrm{L}$ & 0 \\
\hline$Y$ & $\mu \mathrm{g} / \mathrm{L}$ & 0 \\
\hline$Y$ & $\mu g / L$ & 0 \\
\hline$Y$ & $\mu \mathrm{g} / \mathrm{L}$ & 0 \\
\hline$Y$ & $\mu g / L$ & 0 \\
\hline VY & $\mu \mathrm{g} / \mathrm{L}$ & 0 \\
\hline$Y$ & $\mu \mathrm{g} / \mathrm{L}$ & 0 \\
\hline VY & $\mu \mathrm{g} / \mathrm{L}$ & 0 \\
\hline$Y$ & $\mu \mathrm{g} / \mathrm{L}$ & 0 \\
\hline$Y$ & $\mu g / L$ & 0 \\
\hline$Y$ & $\mu g / L$ & 0 \\
\hline$Y$ & $\mu \mathrm{g} / \mathrm{L}$ & 0 \\
\hline$Y$ & $\mu g / L$ & 1 \\
\hline$Y$ & $\mu \mathrm{g} / \mathrm{L}$ & 0 \\
\hline Y & $\mu \mathrm{g} / \mathrm{L}$ & 0 \\
\hline$Y$ & $\mu \mathrm{g} / \mathrm{l}$ & 0 \\
\hline$Y$ & $\mu g / h$ & 0 \\
\hline Y & $\mu \mathrm{g} / \mathrm{L}$ & 0 \\
\hline $\begin{array}{l}\text { JY3 } \\
\text { JY3 }\end{array}$ & $\mathrm{pCi} / \mathrm{L}$ & 0 \\
\hline & $\mathrm{pCi} / \mathrm{mL}$ & 2 \\
\hline
\end{tabular}

\section{WELL HAC 4}

\begin{tabular}{|c|c|c|c|c|c|c|}
\hline$\underline{\text { SRS Coord. }}$ & Lat/Longitude & Screen Zone Elevation & Top of Casing & Casing & Pump & Formation \\
\hline $\begin{array}{l}\text { N72120.3 } \\
561372.0\end{array}$ & $\begin{array}{l}33.285416^{\circ} \mathrm{N} \\
81.645287^{\circ} \mathrm{W}\end{array}$ & 27 & 2 & ; & s & \\
\hline
\end{tabular}

Time: $12: 52$

pH: 4.8

Alkalinity: $0 \mathrm{mg} / \mathrm{L}$

Water temperature: $23.1^{\circ} \mathrm{C}$

Volumes purged: 4.3 well volumes
Sp. conductance: 57

Water evacuated before sampling: $40 \mathrm{gal}$

\section{LABORATORY ANALYSES}

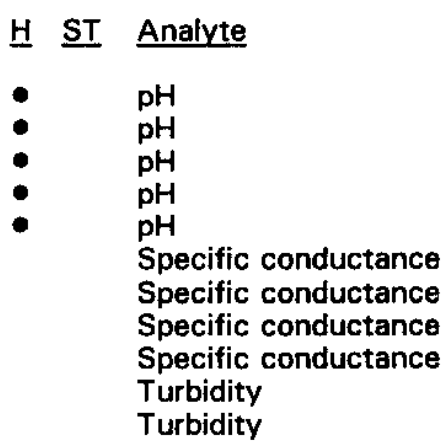

\begin{tabular}{c} 
Result \\
\hline 4.8 \\
5.0 \\
5.2 \\
5.1 \\
5.0 \\
45 \\
46 \\
43 \\
43 \\
$<0.10$ \\
$<0.10$
\end{tabular}

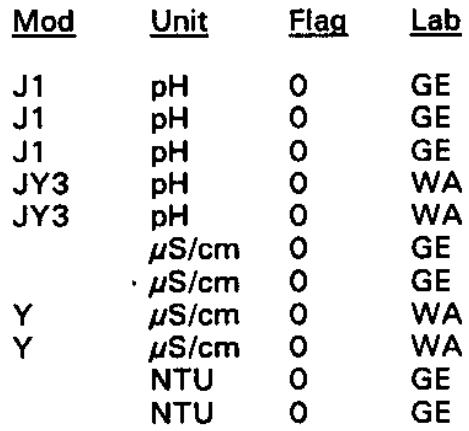

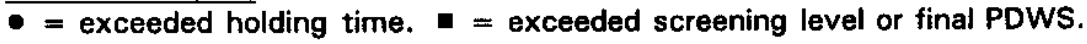


WELL HAC 4 collected on 07/21/94, laboratory analyses (cont.)

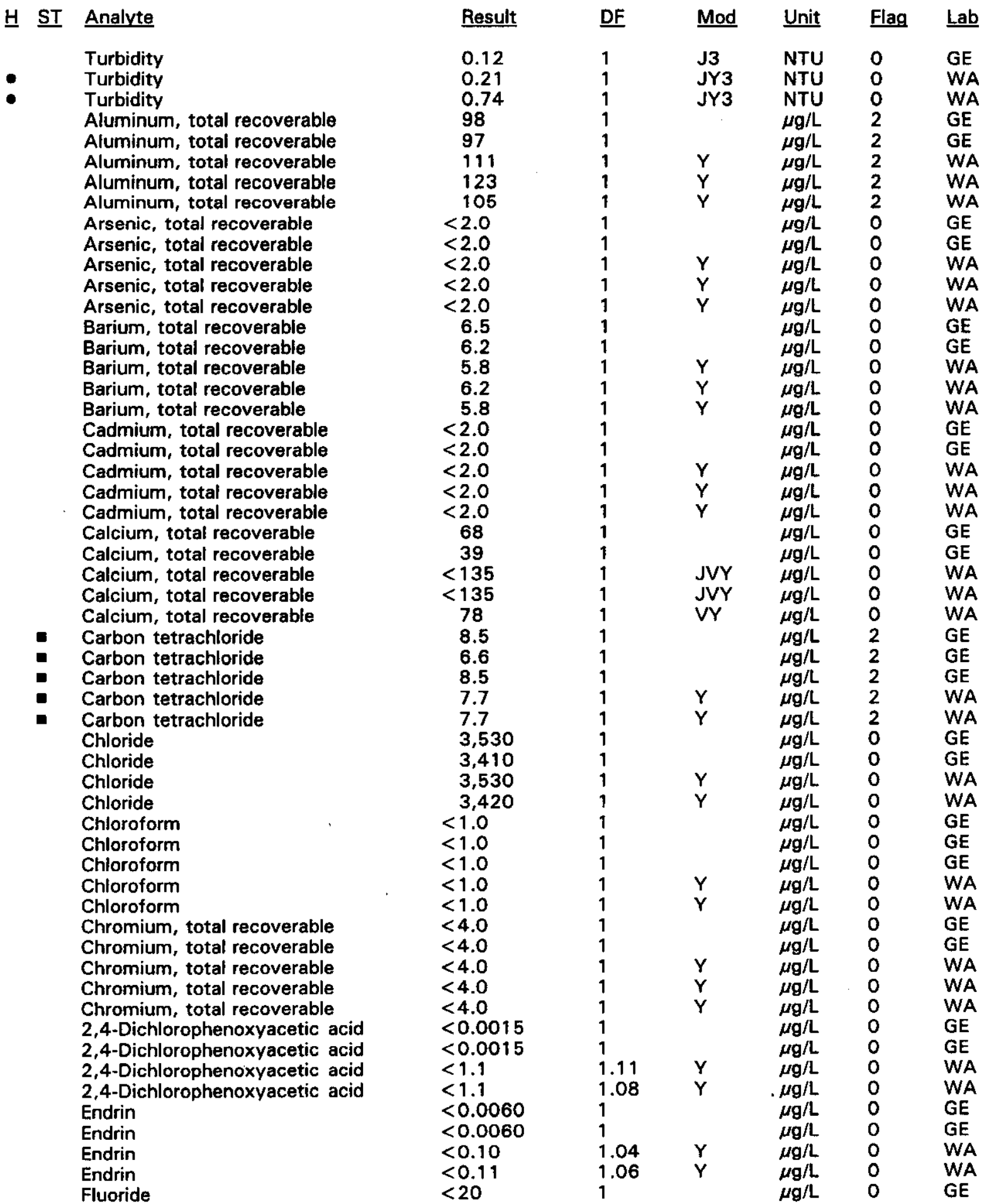

- = exceeded holding time. = exceeded screening level or final PDWS. 
WELL HAC 4 collected on $07 / 21 / 94$, laboratory analyses (cont.)

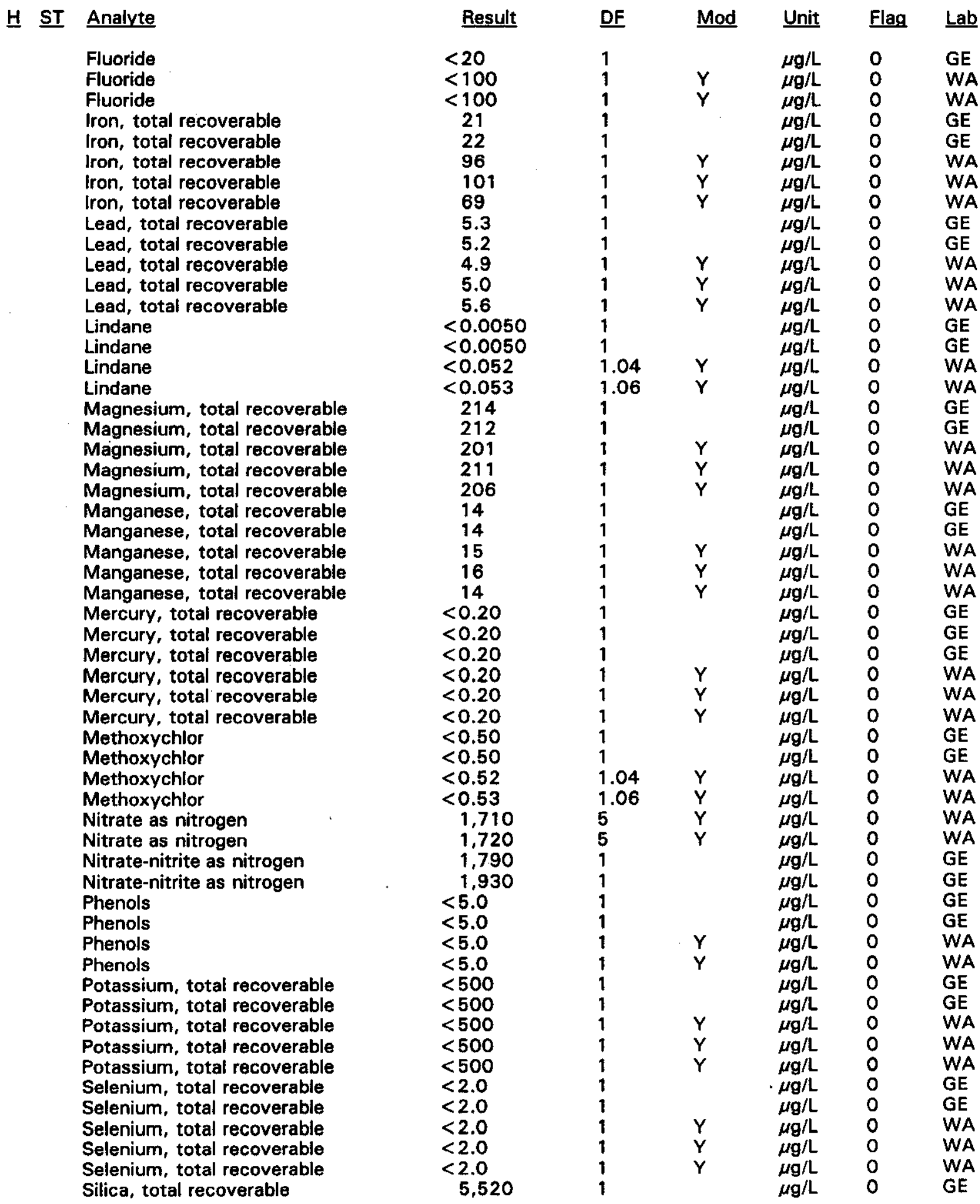

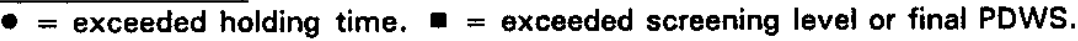


WELL HAC 4 collected on $07 / 21 / 94$, laboratory analyses (cont.)

H ST Analvte

Silica, total recoverable

Silica, total recoverable

Silica, total recoverable

Silica, total recoverable

Silver, total recoverable

Silver, total recoverable

Silver, total recoverable

Silver, total recoverable

Silver, total recoverable

Sodium, total recoverable

Sodium, total recoverable

Sodium, total recoverable

Sodium, total recoverable

Sodium, total recoverable

Sulfate

Sulfate

Sulfate

Sulfate

Tetrachloroethylene

Tetrachloroethylene

Tetrachloroethylene

Tetrachloroethylene

Tetrachloroethylene

Total dissolved solids

Total dissolved solids

Total dissolved solids

Total dissolved solids

Total organic carbon

Total organic carbon

Total organic carbon

Total organic carbon

Total organic halogens

Total organic halogens

Total organic halogens

Total organic halogens

Total phosphates (as P)

Total phosphates (as P)

Total phosphates (as P)

Total phosphates (as P)

Toxaphene

Toxaphene

Toxaphene

Toxaphene

2,4,5-TP (Silvex)

2,4,5-TP (Silvex)

2,4,5-TP (Silvex)

2,4,5-TP (Silvex)

1,1,1-Trichloroethane

1,1,1-Trichloroethane

$1,1,1$-Trichloroethane

$1,1,1$-Trichloroethane

Trichloroethylene

Trichloroethylene

Trichloroethylene

Trichloroethylene

Trichloroethylene

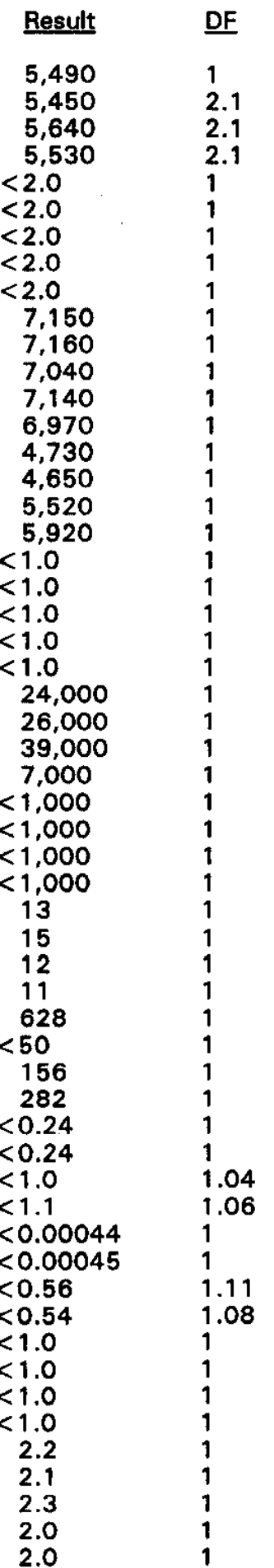

Mod Unit Flag Lab

$\mu \mathrm{g} / \mathrm{L} \quad 0 \quad \mathrm{GE}$

JY3 $\mu \mathrm{g} / \mathrm{L} \quad 0 \quad$ WA

JY3 $\mu g / L \quad 0 \quad W A$

$\begin{array}{llll}Y & \mu g / L & 0 & W A\end{array}$

$\mu g / L \quad 0 \quad G E$

$\mathrm{GE}$

WA

WA

WA

GE

GE

WA

WA

WA

GE

GE

WA

WA

GE

GE

GE

WA

WA

GE

GE

WA

WA

GE

GE

WA

WA

GE

GE

WA

WA

GE

GE

WA

WA

GE

GE

WA

WA

GE

$\mathrm{GE}$

WA

WA

GE

GE

WA

WA

GE

GE

GE

WA

= exceeded holding time. = exceeded screening level or final PDWS. 
WELL HAC 4 collected on $07 / 21 / 94$, laboratory analyses (cont.)

$\underline{H} \underline{\text { ST }}$ Analyte

Gross alpha

Gross alpha

Gross alpha

Gross alpha

Gross alpha

Nonvolatile beta

Nonvolatile beta

Nonvolatile beta

Nonvolatile beta

Nonvolatile beta

- Tritium

- Tritium

Tritium

Tritium

Tritium
Result

$1.9 E+00$

$1.4 \mathrm{E}+00$

$3.1 E+00$

$2.5 \mathrm{E}+00$

$3.6 E+00$

$1.6 \mathrm{E}+00$

9.0E-01

$1.2 E+00$

$3.0 E+00$

$1 . \mathrm{OE}+\mathrm{OO}$

3. $3 E+01$

$3.2 E+01$

$3.0 \mathrm{E}+01$

3. $\mathrm{OE}+01$

$3.1 E+01$ $\underline{\text { DF }}$

Mod

53

J3

UI

UI

UI

UI

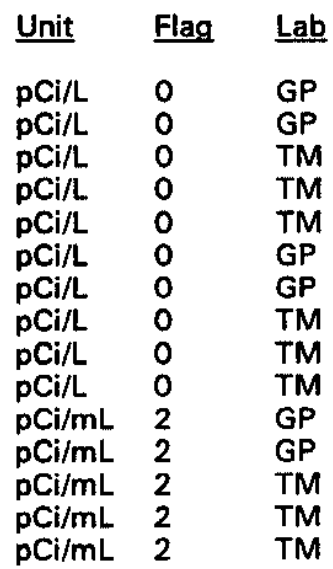

- = exceeded holding time. $\|=$ exceeded screening level or final PDWS. 
WSRC-TR-94-0491

Unclassiffed

\section{Appendix E}

\section{Data Quality/Usability Assessment}


WSRC-TR-94-0491

Unclassified

THIS PAGE LEFT BLANK INTENTIONALLY. 


\section{Data Quality/Usability Assessment}

Quality assurance/quality control (QAVQC) procedures relating to accuracy and precision of analyses performed on groundwater samples are followed in the field and laboratory and are reviewed prior to publication of results. The review by the Environmental Protection Department/Environmental Monitoring Section (EPD/EMS) of the volume of analytical data acquired each quarter and presented in various reports is an ongoing process; its review of the QA/QC data cannot be completed in time to meet the deadlines for the reports required by the Resource Conservation and Recovery Act and associated regulations. Other site and regulatory personnel can obtain further information on the data quality and usability in a variety of ways, including those described below.

\section{Data Qualification}

The contract laboratories continually assess their own accuracy and precision according to U.S. Environmental Protection Agency (EPA) guidelines. They submit sample- or batch-specific QA QC information either at the same time as analytical results or in quarterly summaries. Properly defined and used result modifiers (also referred to as qualifiers) can be a key component in assessing data usability. Result modifiers designed by EPD/EMS and used by the primary laboratories are presented in Appendix D.

\section{Assessment of Accuracy of the Data}

Accuracy, or the nearness of the reported result to the true concentration of a constituent in a sample, can be assessed in several ways.

A laboratory's general accuracy can be judged by analysis of results obtained from known samples. The non-radionuclide contract laboratories analyze commercial reference samples every quarter at EPD/EMS' request. The results of these analyses are presented in the EPD/EMS groundwater monitoring quarterly reports. The primary laboratories also seek or maintain state certification by participating periodically in performance studies; reference samples and analysis of results are provided by EPA. Results of these studies also are published in the EPD/EMS quarterly reports.

Analysis of blanks provides a tool for assessing the accuracy of both sampling and laboratory analysis. Results for all field blanks for the quarter can be found in the EPD/EMS quarterly reports. Any field or laboratory blanks that exceed established minimums are identified in the same reports, in tables associating them with groundwater samples analyzed in the same batches.

Surrogates, organic compounds similar in chemical behavior to the compounds of interest but not normally found in environmental samples, are used to monitor the effect of the matrix on the accuracy of analyses for organic parameters. For example, for analyses of volatile organics by EPA Method 8240 , three surrogate compounds are added to all samples and blanks in each analytical batch. In analyses of semivolatile organics, three acid compounds and three basel neutral compounds are used. Two surrogates are used in organochlorine pesticides analyses. Percent recoveries for surrogate analyses are calculated by laboratory personnel, reported to EPD/EMS, reviewed, and entered into the database, but they are not published. If recoveries are not within specified limits, the laboratory is expected to reanalyze the samples or attach qualifiers to the data identifying the anomalous results. 
Sample-specific accuracy for both organic and inorganic parameters can be assessed by examination of matrix spike/matrix spike duplicate results. A sample is analyzed unspiked to determine a baseline set of values. A second portion of the sample is spiked with known concentrations of compounds appropriate to the analyses being performed, typically five volatile organic compounds for volatile organics analyses, eleven semivolatile compounds for semivolatiles, six pesticide compounds for pesticides, all metals for metals analyses by SW-846 methods (EPA, 1986), and a known quantity of cyanide for cyanide analysis. The percentage of the spike compound that is recovered (i.e., measured in excess of the value obtained for the unspiked sample) is a direct measure of analytical accuracy. EPA requires matrix spike/matrix spike duplicates to be run at least once per 20 samples of similar matrix.

Matrix spike/matrix spike duplicate results are reported to EPD/EMS but are not published. For organic compounds, according to EPA guidelines, no action is taken on the basis of matrix spike/ matrix spike duplicate data alone (i.e., no result modifiers are assigned solely on the basis of matrix spike results); however, the results can indicate if a laboratory is having a systematic problem in the analysis of one or more analytes.

In the case of inorganic compounds, such as metals, the matrix spike sample analysis provides information about the effect of each sample matrix on the digestion and measurement methodology. Data qualifiers assigned by the laboratories on the basis of the percentage of spike recovery are reported in the published results tables.

\section{Assessment of Precision}

Precision of the analyses, or agreement of a set of replicate results among themselves, is assessed through the use of duplicates initiated by the laboratory and blind replicates provided by EPD/EMS. The results of duplicate and replicate analyses are presented in those results tables of the quarterly reports which report only one quarter of data, usually during first, second, and third quarters. Duplicate and replicate results are not presented in results tables that report more than one quarter of data, generally provided in fourth quarter reports. In this case, the results tables instead present only the highest result for each analyte for each quarter of the year.

The laboratories assess precision by calculating the relative percent difference (RPD) for each pair of laboratory-initiated duplicate results. One of the contract laboratories uses a data qualifier (J3) to modify metals analyses when the RPD for laboratory duplicates is greater than 20 percent.

Additional statistical comparisons of laboratory duplicate and blind replicate results, both intraand interlaboratory, are presented in the EPD/EMS quarterly reports. The calculation used for these reports is the mean relative difference (MRD) which is similar to EPA's RPD except that the MRD is the average of all the RPD values from one laboratory for each compound (intralaboratory MRD) or all the RPD values from all laboratories for each compound (interlaboratory $M R D$ ), during one quarter. Because detection limits may vary among samples, the MRD requires calculation of a reference detection limit, which is the detection limit at the 90th percentile of the array of limits in the population of all duplicate and replicate analyses for a given analyte during a particular quarter. The MRD is not method-specific.

\section{Method-Specific Accuracy and Precision}

The contract laboratories' EPA-approved laboratory procedures include QAVC requirements as an integral part of the methods. Thus, knowledge of the method used in obtaining data is an important component of determining data usability. EPA has conducted extensive research and 
development on the methods approved for the analysis of water and waste water; information on the accuracy and precision of a method is available from EPA publications, as is full information on required QAQC procedures. A listing of the methods used by the primary laboratories during fourth quarter 1993 is given below along with the source for the method description. Many, if not all, of these sources include presentations of representative accuracy and precision results.

\section{Methods Used by the Contract Laboratories}

\begin{tabular}{|c|c|c|}
\hline Method & Used to Analyze & Source \\
\hline EPA120.1 & Specific conductance & EPA EMSL, 1983 \\
\hline EPA150.1 & $\mathrm{pH}$ & EPA EMSL, 1983 \\
\hline EPA160.1 & Total dissolved solids & EPA EMSL, 1983 \\
\hline EPA160.2 & Total dissolved solids, total suspended solids & EPA EMSL, 1983 \\
\hline EPA180.1 & Turbidity & EPA EMSL, 1983 \\
\hline EPA200.7 & Metals & EPA EMSL, 1983 \\
\hline EPA204.2 & Antimony & EPA EMSL, 1983 \\
\hline EPA206.2 & Arsenic & EPA EMSL, 1983 \\
\hline EPA239.2 & Lead & EPA EMSL, 1983 \\
\hline EPA245.1 & Mercury & EPA EMSL, 1983 \\
\hline EPA270.2 & Selenium & EPA EMSL, 1983 \\
\hline EPA279.2 & Thallium & EPA EMSL, 1983 \\
\hline EPA300.0 & Chloride, nitrite, sulfate & EPA EMSL, 1991 \\
\hline EPA310.1 & Alkalinity & EPA EMSL, 1983 \\
\hline EPA325.2 & Chloride & EPA EMSL, 1983 \\
\hline EPA335.3 & Cyanide & EPA EMSL, 1983 \\
\hline EPA340.2 & Fluoride & EPA EMSL, 1983 \\
\hline EPA353.1 & Nitrogen, nitrate-nitrite & EPA EMSL, 1983 \\
\hline EPA353.2 & Nitrogen, nitrate, nitrite, or combined & EPA EMSL, 1983 \\
\hline EPA365.1 & Phosphorus, all forms (reported as total phosphates) & EPA EMSL, 1983 \\
\hline EPA365.2 & Phosphorus, all forms (reported as total phosphates) & EPA EMSL, 1983 \\
\hline EPA376.2 & Sulfide & EPA EMSL, 1983 \\
\hline EPA413.1 & Oil \& grease & EPA EMSL, 1983 \\
\hline EPA415.1 & $\begin{array}{l}\text { Dissolved organic carbon, total inorganic carbon, total organic } \\
\text { carbon }\end{array}$ & EPA EMSL, 1983 \\
\hline EPA418.1 & Total petroleum hydrocarbons & EPA EMSL, 1983 \\
\hline EPA420.2 & Phenols & EPA EMSL, 1983 \\
\hline EPA900.0 & Gross alpha, nonvolatile beta & EPA EMSL, 1980 \\
\hline EPA900.1 & Total alpha-emitting radium & EPA EMSL, 1980 \\
\hline EPA906.0 & Tritium & EPA EMSL, 1980 \\
\hline EPA6010 & Metals & EPA, 1986 \\
\hline EPA7041 & Antimony & EPA, 1986 \\
\hline EPA7060 & Arsenic & EPA, 1986 \\
\hline EPA7421 & Lead & EPA, 1986 \\
\hline EPA7470 & Mercury & EPA, 1986 \\
\hline EPA7740 & Selenium & EPA, 1986 \\
\hline EPA7841 & Thallium & EPA, 1986 \\
\hline EPA8010 & Chlorinated volatile organics & EPA, 1986 \\
\hline EPA8080 & Organochlorine pesticides and PCBs & EPA, 1986 \\
\hline EPA8150 & Chlorinated herbicides & EPA, 1986 \\
\hline EPA8240 & GCMS volatiles & EPA, 1986 \\
\hline EPA8270 & GCMS semivolatiles & EPA, 1986 \\
\hline EPA8280 & Dioxins and furans & EPA, 1986 \\
\hline & Cvanide & EPA, 1986 \\
\hline EPA9020 & Total organic halogens & EPA, 1986 \\
\hline EPA9020A & Total organic halogens & EPA, 1986 \\
\hline EPA9030 & Sulfide & EPA, 1986 \\
\hline
\end{tabular}


Method

EPA9060

\section{Used to Analyze}

Dissolved organic carbon, total inorganic carbon, total organic carbon $\underline{\text { Source }}$

EPA, 1986

An example of available method-specific QAVQ information is that for the analysis of metals by EPA Method 6010/200.7 (EPA, 1986/EPA EMSL, 1983). The primary laboratories, General Engineering Laboratories (GE) and Roy F. Weston, Inc. (Weston), use this inductively coupled plasma (ICP) atomic emission spectrometric method.

The following precision and accuracy data are based on the experience of seven laboratories that applied the ICP technique to acid-distilled water matrices that had been spiked with various metal concentrates. (Note: Not all seven laboratories analyzed all 14 elements.) The references give results for samples having three concentration ranges; the results here are for samples having the lowest values, similar to actual groundwater results for SRS.

ICP Precision and Accuracy Data

\begin{tabular}{ll} 
Element & I \\
\cline { 2 - 2 } Aluminum & 60 \\
Arsenic & 22 \\
Beryllium & 20 \\
Cadmium & 25 \\
Chromium & 10 \\
Cobalt & 20 \\
Copper & 11 \\
Iron & 20 \\
Lead & 24 \\
Manganese & 15 \\
Nickel & 30 \\
Selenium & 6 \\
Vanadium & 70 \\
Zinc & 16
\end{tabular}

22

20

2.5

10

20

11

20

24

15

30

6

70
16 $\begin{array}{ll}\text { True value }(\mu \mathrm{g} / \mathrm{L}) & \begin{array}{l}\text { Mean reported } \\ \text { value }(\mathrm{gg} / \mathrm{L})\end{array}\end{array}$

60

62

19

$20 \quad 9.8$

$2.9 \quad 16$

$10 \quad 18$

$20 \quad 4.1$

1140

19

$30 \quad 32$

$15 \quad 6.7$

$28 \quad 11$

$8.5 \quad 42$

$69-2.9$

$19 \quad 45$

45
Mean percent $\underline{\mathrm{RSD}}^{\mathrm{a}}$

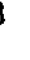

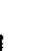

40

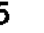

7

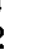

\footnotetext{
a Relative standard deviation. In EPA (1986), the column heading is Mean Standard Deviation (\%).
}

As another example, EPA Method 601/8010 (EPA, 1991/EPA, 1986) is used by both GE and Weston for analyses of halogenated volatile organics. In the presentation of the method in both references, the following table gives method-specific accuracy and precision as functions of concentration. Contract laboratories are expected to achieve or at least approach these limits.

\section{Accuracy and Precision as Functions of Concentration for EPA Method 601/8010}

$\begin{array}{llll}\text { Parameter } & \begin{array}{l}\text { Accuracy as } \\ \text { recovery, } X^{\prime a}(\mu g / L)\end{array} & \begin{array}{l}\text { Single analyst } \\ \text { precision }(\mu g / L)^{b}\end{array} & \begin{array}{l}\text { Overall } \\ \text { precision }(\mu g / L)^{c}\end{array} \\ \text { Bromodichloromethane } & 1.12 C-1.02^{d} & 0.11 \bar{X}+0.04^{\mathrm{d}} & 0.20 \bar{X}+1.00 \\ \text { Bromoform } & 0.96 C-2.05 & 0.12 \bar{X}+0.58 & 0.21 \bar{X}+2.41 \\ \text { Bromomethane } & 0.76 C-1.27 & 0.28 \bar{X}+0.27 & 0.36 \bar{X}+0.94 \\ \text { Carbon tetrachloride } & 0.98 C-1.04 & 0.15 \bar{X}+0.38 & 0.20 \bar{X}+0.39 \\ \text { Chlorobenzene } & 1.00 C-1.23 & 0.15 \bar{X}-0.02 & 0.18 \bar{X}+1.21 \\ \text { Chloroethane } & 0.99 C-1.53 & 0.14 \bar{X}-0.13 & 0.17 \bar{X}+0.63 \\ \text { 2-Chloroethyl vinyl ether }{ }^{f} & 1.00 C & 0.20 \bar{X} & 0.35 \bar{X}\end{array}$


Parameter

Chloroform

Chloromethane

Dibromochloromethane

1,2-Dichlorobenzene

1,3-Dichlorobenzene

1,4-Dichlorobenzene

1,1-Dichloroethane

1,2-Dichloroethane

1,1-Dichloroethene

trans-1,2-Dichloroethene

Dichloromethane

(Methylene chloride)

1,2-Dichloropropane ${ }^{f}$

cis-1,3-Dichloropropene $f$

trans-1,3-Dichloropropene ${ }^{f}$

1,1,2,2-Tetrachloroethane

Tetrachloroethylene

1,1,1-Trichloroethane

1,1,2-Trichloroethane

Trichloroethylene

Trichlorofluoromethane

Vinyl chloride
Accuracy as

recovery, $X^{\prime} \quad(\mu g / L)$

$0.93 C-0.39$

$0.77 C+0.18$

$0.94 C+2.72$

$0.93 C+1.70$

$0.95 C+0.43$

$0.93 C-0.09$

$0.95 C-1.08$

$1.04 C-1.06$

$0.98 C-0.87$

$0.97 C-0.16$

$0.91 C-0.93$

$1.00 \mathrm{C}$

$1.00 \mathrm{C}$

$1.00 \mathrm{C}$

$0.95 C+0.19$

$0.94 C+0.06$

$0.90 C-0.16$

$0.86 C+0.30$

$0.87 C+0.48$

$0.89 \mathrm{C}-0.07$

$0.97 C-0.36$
Single analyst

precision ( $\mu \mathrm{g} / \mathrm{L}$ )

$0.13 \bar{X}+0.15$

$0.28 \bar{X}-0.31$

$0.11 \bar{X}+1.10$

$0.20 \bar{X}+0.97$

$0.14 \bar{X}+2.33$

$0.15 \bar{X}+0.29$

$0.09 \bar{X}+0.17$

$0.11 \bar{X}+0.70$

$0.21 \bar{X}-0.23$

$0.11 \bar{X}+1.46$

$0.11 \bar{X}+0.33$

$0.13 \bar{X}$

$0.18 X$

$0.18 \bar{X}$

$0.14 \bar{X}+2.41$

$0.14 X+0.38$

$0.15 \bar{X}+0.04$

$0.13 \bar{X}-0.14$

$0.13 \bar{X}-0.03$

$0.15 \bar{X}+0.67$

$0.13 \bar{X}+0.65$
Overall

precision $(\mu \mathrm{g} / \mathrm{L})$

$0.19 \bar{X}-0.02$

$0.52 \bar{X}+1.31$

$0.24 \bar{X}+1.68$

$0.13 \bar{X}+6.13$

$0.26 \bar{X}+2.34$

$0.20 \bar{X}+0.41$

$0.14 \bar{X}+0.94$

$0.15 \bar{X}+0.94$

$0.29 \bar{X}-0.40$

$0.17 \bar{X}+1.46$

$0.21 \bar{X}+1.43$

$0.23 \bar{X}$

$0.32 \bar{X}$

$0.32 \bar{X}$

$0.23 \bar{X}+2.79$

$0.18 \bar{X}+2.21$

$0.20 \bar{X}+0.37$

$0.19 \bar{X}+0.67$

$0.23 \bar{X}+0.30$

$0.26 \bar{X}+0.91$

$0.27 \bar{X}+0.40$

a $X^{\prime}=$ expected recovery for one or more measurements of a sample containing a concentration of $C$, in $\mu \mathrm{g} / \mathrm{L}$.

b Expected single analyst standard deviation of measurements.

c Expected intertaboratory standard deviation of measurements.

d $C=$ true value for the concentration, in $\mu \mathrm{g} / \mathrm{L}$.

e $\bar{X}=$ average recovery found for measurements of samples containing a concentration of $C$, in $\mu g / L$.

$f$ Estimates based on performance of a single laboratory.

\section{References}

EPA (U.S. Environmental Protection Agency), 1986. Test Methods for Evaluating Solid Waste (SW-846), Volumes IA-IC. Washington, DC.

EPA (U.S. Environmental Protection Agency), 1991. Guidelines Establishing Test Procedures for the Analysis of Pollutants, Code of Federal Regulations, Title 40, Part 136, Appendix A. Washington, DC.

EPA EMSL (U.S. Environmental Protection Agency, Environmental Monitoring and Systems Laboratory), 1980. Prescribed Procedures for Measurement of Radioactivity in Drinking Water, EPA-600/4-80-032. Cincinnati, $\mathrm{OH}$.

EPA EMSL (U.S. Environmental Protection Agency, Environmental Monitoring and Systems Laboratory), 1983. Methods for Chemical Analysis of Water and Wastes. Cincinnati, $\mathrm{OH}$.

EPA EMSL (U.S. Environmental Protection Agency, Environmental Monitoring and Systems Laboratory), 1991. Test Method, The Determination of Inorganic Anions in Water by Ion Chromatography-Method 300.0. Cincinnati, $\mathrm{OH}$. 
WSRC-TR-94-0491

Unclassified

THIS PAGE LEFT BLANK INTENTIONALLY. 